\title{
Final Report on Initial Samples Supplied by LLNL for Task 3.3 Binder Burnout and Sintering Schedule Optimisation
}

P. Walls

\section{January 4, 1999}

U.S. Department of Energy

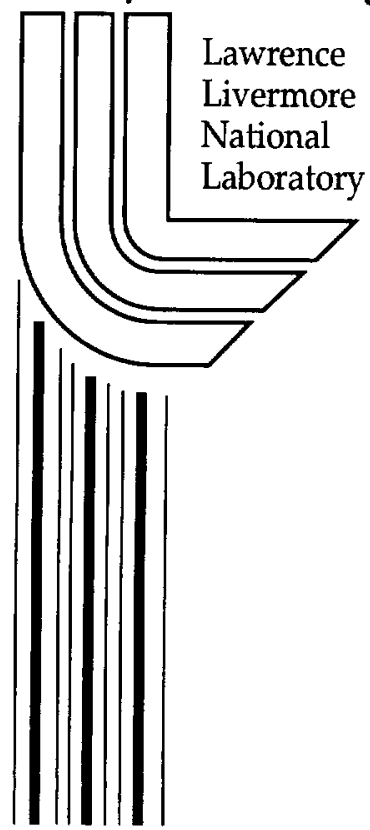




\section{DISCLAIMER}

This document was prepared as an account of work sponsored by an agency of the United States Government. Neither the United States Government nor the University of California nor any of their employees, makes any warranty, express or implied, or assumes any legal liability or responsibility for the accuracy, completeness, or usefulness of any information, apparatus, product, or process disclosed, or represents that its use would not infringe privately owned rights. Reference herein to any specific commercial product, process, or service by trade name, trademark, manufacturer, or otherwise, does not necessarily constitute or imply its endorsement, recommendation, or favoring by the United States Government or the University of California. The views and opinions of authors expressed herein do not necessarily state or reflect those of the United States Government or the University of California, and shall not be used for advertising or product endorsement purposes.

This work was performed under the auspices of the U.S. Department of Energy by the University of California, Lawrence Livermore National Laboratory under Contract No. W-7405-Eng-48.

This report has been reproduced directly from the best available copy.

Available electronically at http://www.doe.gov/bridge

Available for a processing fee to U.S. Department of Energy

and its contractors in paper from

U.S. Department of Energy

Office of Scientific and Technical Information

P.O. Box 62

Oak Ridge, TN 37831-0062

Telephone: (865) 576-8401

Facsimile: (865) 576-5728

E-mail: reports@adonis.osti.gov

Available for the sale to the public from

U.S. Department of Commerce

National Technical Information Service

5285 Port Royal Road

Springfield, VA 22161

Telephone: (800) 553-6847

Facsimile: (703) 605-6900

E-mail: orders@ntis.fedworld.gov

Online ordering: http://www.ntis.gov/ordering.htm

OR

Lawrence Livermore National Laboratory

Technical Information Department's Digital Library

http://www.llnl.gov/tid/Library.html 


\title{
Onsto \\ materials division
}

Final Report on Initial Samples Supplied by LLNL For Task 3.3 Binder Burnout and Sintering Schedule Optimisation

\author{
Dr P Walls
}

4 January, 1999

\section{R99m003}

Australian Nuclear Science and Technology Organisation

Private Mail Bag 1, Menai, NSW 2234 


\section{Final Report on Initial Samples Supplied by LLNL For Task 3.3 Binder Burnout and Sintering Schedule Optimisation}

DATE ISSUED:

4 January, 1999

ISSUED TO:

Lawrence Livermore National Laboratory

REPORT NUMBER:

JOB NUMBER

R99m003

$713 \mathrm{~m}$

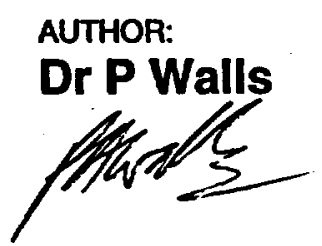

APPROVED BY:
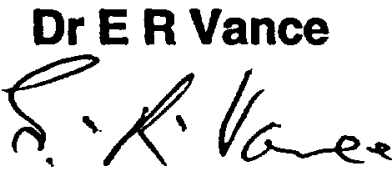

Australian Nuclear Science and Technology Organisation Postal Address: Private Mail Bag 1, Menai, NSW 2234, Australia Materials Division: Telephone +612 97173265 Facsimile +61 295437179 


\title{
Final Report on Initial Samples Supplied by LLNL for Task 3.3 Binder Burnout and Sintering Schedule Optimisation
}

\author{
Dr. Philip Walls, \\ ANSTO Materials Division
}

\section{Summary}

Sixteen of the twenty-one samples have been investigated using the scanning laser dilatometer. This includes all three types of samples with different preparation routes and organic content. Cracks were observed in all samples, even those only heated to $300^{\circ} \mathrm{C}$. It was concluded that the cracking was occurring in the early part of the heat treatment before the samples reached $300^{\circ} \mathrm{C}$. Increase in the rate of dilation of the samples occurred above $170^{\circ} \mathrm{C}$ which coincided with the decomposition of the binder/wax additives as determined by differential thermal analysis. A comparison was made with SYNROC C material (Powder Run 143), samples of which had been CIPed and green machined to a similar diameter and thickness as the $\varnothing 89 \mathrm{~mm}$ SRTC pucks. These samples contained neither binder nor other organic processing aids and had been kept in the same desiccator as the SRTC samples. The CIPed Synroc C samples sintered to high density with zero cracks.

As the cracks made up only a small contribution to the change in diameter of the sample compared to the sintering shrinkage, useful information could still be gained from the runs. The sintering curves showed that there was much greater shrinkage of the Type III samples containing only the 5\% PEG binder compared to the Type I which contained polyolefin wax as processing aid. Slight changes in gradient of the sintering curve were observed, however, due to the masking effect of the cracking, full analysis of the sintering kinetics cannot be conducted.

Even heating the samples to $300^{\circ} \mathrm{C}$ at 1.0 or $0.5^{\circ} \mathrm{C} / \mathrm{min}$ could not prevent crack formation. This indicated that heating rate was not the critical parameter causing cracking of the samples.

Sectioning of green bodies revealed the inhomogeneous nature of the binder/lubricant distribution in the samples. Increased homogeneity would reduce the amount of binder/lubricant required, which should in turn, reduce the degree of cracking observed during heating to the binder burnout temperature.

A combination of; 1) use of a higher forming pressure, 2) reduction of organics content, 3) improvement in the distribution of the organic wax and binder components throughout the green body, could possibly alleviate cracking. Ultrasonic emulsification of the binder and wax with a small quantity of water prior to adding to the ball or attrition mill is advised to ensure more even distribution of the wax / binder system. This would also reduce the proportion of organic additives required. 
The binder burnout stage of the operation must first be optimised (ie. production of pucks with no cracks) prior to optimisation of the sintering stage.

Table 1. Sample Nomenclature and Description

\begin{tabular}{|c|c|c|c|c|c|c|c|}
\hline Type & $\begin{array}{c}\text { Precursor } \\
\text { Calcined }\end{array}$ & $\begin{array}{c}\text { All components } \\
\text { Calcined }\end{array}$ & $\begin{array}{c}\text { Wet Ball- } \\
\text { Milled }\end{array}$ & $\begin{array}{c}\text { Attrition } \\
\text { Milled }\end{array}$ & $\begin{array}{c}\text { PEG } \\
\mathbf{8 0 0 0}\end{array}$ & $\begin{array}{c}\text { WAX } \\
\text { A-12 }\end{array}$ & $\begin{array}{c}\text { Samples } \\
\text { LLL series }\end{array}$ \\
\hline I & Yes & No & Yes & No & $\mathbf{5}$ & $\mathbf{0}$ & $\mathbf{1 5 3 , 2 3 3 , 2 3 4 , 2 3 5 , 2 3 6 , 2 3 7}$ \\
\hline II & No & Yes & Yes & No & $\mathbf{5}$ & $\mathbf{0}$ & $\mathbf{1 6 1 , 1 6 2 , 1 6 3 , 2 3 8 , 2 3 9 , 2 4 0 , 2 4 1}$ \\
\hline III & No & No & No & Yes & $\mathbf{5}$ & $\mathbf{3}$ & $\mathbf{1 6 7 , 1 6 8 , 1 6 9 , 2 4 2 , 2 4 3 , 2 4 4 , 2 4 5 , 2 4 6}$ \\
\hline
\end{tabular}

(NB numbers in bold indicate samples which have been investigated)

\section{Heat Treatments}

Table 2 shows the heat treatments used. Two samples were heat treated in the majority of runs, with sample dimensions being monitored using separate laser sensors. The samples were placed on $100 \times 100 \times 0.3 \mathrm{~mm}$ thick platinum sheets, resting on $\varnothing 120 \times 3 \mathrm{~mm}$ thick alumina plates.

\section{Scanning Laser Dilatometer Runs}

The results will be described in terms of SLD run numbers, which also indicate the date the run was performed. Table 3 shows the heating patterns used with each sample type, and Table $4 \& 5$ summarise the dimensional changes, volume shrinkage and density increase of the samples. Figures 1 to 4 summarise the results from Table 4 \& 5 .

Run SLD A981020 : CIPed, zero organics content, ANSTO Synroc C (Powder Run 143) sample for comparison. No cracking was observed.

Run SLD A981021 : Run using standard baseline heat treatment. Type I sample containing 5\% PEG only. The sample cracked, see Figure 11.

Run SLD A981022 : Run using standard baseline heat treatment. LLL242 (Type III) sample containing 5\% PEG $+3 \%$ wax. It was thought that the cracking of the previous sample might have been due to its processing conditions. Hence a Type III sample was run for comparison. The sample also cracked, but in different manner to the Type I samples, see Figure 12.

It was then thought that the cracks in the sample were possibly caused by a high moisture content in the green body resulting from pick up during shipment of samples from the US to Australia and during storage at ANSTO, even though the pucks had been triple bagged by LLL in heat shrink plastic prior to shipping and had been stored in a desiccator since arriving at ANSTO. It was decided to determine the moisture content of the samples. Type I a Type III and a Synroc C (Run 143) sample were placed in an oven at $50^{\circ} \mathrm{C}$ for $20 \mathrm{hrs}$. The moisture contents were deduced from the observed weight losses and are shown in Table 5. 
Table 2 Summary of Heat Treatments

LLLHT001

\begin{tabular}{|c|c|c|c|c|}
\hline Step & $\begin{array}{c}\text { Temp } \\
\text { No. }\end{array}$ & $\begin{array}{c}\text { Rate } \\
{ }^{\circ} \mathrm{C}\end{array}$ & $\begin{array}{c}\text { Time } \\
\left({ }^{\circ} \mathrm{C} / \mathrm{min}\right)\end{array}$ & $\begin{array}{c}\text { Cum. } \\
\text { Time } \\
(\mathrm{min})\end{array}$ \\
\hline 0 & 20 & 0 & 0 & 0 \\
1 & 300 & 3 & 93 & 93 \\
2 & 300 & 0 & 120 & 213 \\
3 & 1350 & 2.5 & 420 & 633 \\
4 & 1350 & 0 & 240 & 873 \\
5 & 20 & 5 & 266 & 1139 \\
\hline
\end{tabular}

\section{LLLHT003}

\begin{tabular}{|c|c|c|c|c|}
\hline $\begin{array}{l}\text { Step } \\
\text { No. }\end{array}$ & $\begin{array}{c}\text { Temp } \\
{ }^{\circ} \mathrm{C}\end{array}$ & $\begin{array}{c}\text { Rate } \\
\left.\text { ( }{ }^{\circ} \mathrm{C} / \mathrm{min}\right)\end{array}$ & $\begin{array}{l}\text { Time } \\
\text { (min) }\end{array}$ & $\begin{array}{l}\text { Cum. } \\
\text { Time } \\
\text { (min) }\end{array}$ \\
\hline 0 & 20 & 0 & 0 & 0 \\
\hline 1 & 300 & 3 & 93 & 93 \\
\hline 2 & 300 & 0 & 120 & 213 \\
\hline 3 & 1350 & 12.5 & 84 & 297 \\
\hline 4 & 1350 & 0 & 240 & 537 \\
\hline 5 & 20 & 5 & 266 & 803 \\
\hline
\end{tabular}

LLLHT005

\begin{tabular}{|c|c|c|c|c|}
\hline Step & Temp & Rate & Time & $\begin{array}{c}\text { Cum. } \\
\text { Time } \\
(\text { No. }\end{array}$ \\
\hline 0 & ${ }^{\circ} \mathrm{C}$ & $\left({ }^{\circ} \mathrm{C} / \mathrm{min}\right)$ & $(\mathrm{min})$ & 0 \\
1 & 300 & 0 & 0 & 0 \\
2 & 300 & 0 & 280 & 280 \\
3 & 20 & 5 & 56 & 400 \\
\hline
\end{tabular}

LLLHT007

\begin{tabular}{|c|c|c|c|c|}
\hline $\begin{array}{l}\text { Step } \\
\text { No. }\end{array}$ & $\begin{array}{c}\text { Temp } \\
{ }^{\circ} \mathrm{C}\end{array}$ & $\begin{array}{c}\text { Rate } \\
\left({ }^{\circ} \mathrm{C} / \mathrm{min}\right)\end{array}$ & $\begin{array}{l}\text { Time } \\
\text { (min) }\end{array}$ & $\begin{array}{l}\text { Cum. } \\
\text { Time } \\
\text { (min) }\end{array}$ \\
\hline 0 & 20 & 0 & 0 & 0 \\
\hline 1 & 300 & 0.5 & 560 & 560 \\
\hline 2 & 300 & 0 & 120 & 680 \\
\hline 3 & 20 & 5 & 56 & 736 \\
\hline
\end{tabular}

LLLHT002

\begin{tabular}{|c|c|c|c|c|}
\hline Step & $\begin{array}{c}\text { Temp } \\
\text { No. }\end{array}$ & $\begin{array}{c}\text { Rate } \\
{ }^{\circ} \mathbf{C}\end{array}$ & $\begin{array}{c}\text { Time } \\
\left({ }^{\circ} \mathrm{C} / \mathrm{min}\right)\end{array}$ & $\begin{array}{c}\text { Cum. } \\
\text { Time } \\
(\mathbf{m i n})\end{array}$ \\
\hline 0 & 20 & 0 & 0 & 0 \\
1 & 300 & 3 & 93 & 93 \\
2 & 300 & 0 & 120 & 213 \\
3 & 1350 & 5 & 210 & 423 \\
4 & 1350 & 0 & 240 & 663 \\
5 & 20 & 5 & 266 & 929 \\
\hline
\end{tabular}

\section{LLLHT004}

\begin{tabular}{|c|c|c|c|c|}
\hline $\begin{array}{l}\text { Step } \\
\text { No. }\end{array}$ & $\begin{array}{c}\text { Temp } \\
{ }^{\circ} \mathrm{C}\end{array}$ & $\begin{array}{c}\text { Rate } \\
\left({ }^{\circ} \mathrm{C} / \mathrm{min}\right)\end{array}$ & $\begin{array}{l}\text { Time } \\
\text { (min) }\end{array}$ & $\begin{array}{l}\text { Cum. } \\
\text { Time } \\
\text { (min) }\end{array}$ \\
\hline 0 & 20 & 0 & 0 & 0 \\
\hline 1 & 300 & 3 & 93 & 93 \\
\hline 2 & 300 & 0 & 60 & 153 \\
\hline 3 & 20 & 5 & 56 & 209 \\
\hline
\end{tabular}

LLLHT008

\begin{tabular}{|c|c|c|c|c|}
\hline $\begin{array}{l}\text { Step } \\
\text { No. }\end{array}$ & $\begin{array}{c}\text { Temp } \\
{ }^{\circ} \mathrm{C}\end{array}$ & $\begin{array}{c}\text { Rate } \\
\left({ }^{\circ} \mathrm{C} / \mathrm{min}\right)\end{array}$ & $\begin{array}{l}\text { Time } \\
\text { (min) }\end{array}$ & $\begin{array}{l}\text { Cum. } \\
\text { Time } \\
\text { (min) }\end{array}$ \\
\hline 0 & 20 & 0 & 0 & $\overline{0}$ \\
\hline 1 & 110 & 3 & 30 & 30 \\
\hline 2 & 110 & 0 & 60 & 90 \\
\hline 3 & 300 & 3 & 63 & 153 \\
\hline 4 & 300 & 0 & 60 & 213 \\
\hline 5 & 1350 & 5 & 210 & 423 \\
\hline 6 & 1350 & 0 & 240 & 663 \\
\hline 7 & 20 & 5 & 266 & 929 \\
\hline
\end{tabular}


Table 3. Scanning Laser Dilatometer Runs

\begin{tabular}{|c|c|c|c|c|}
\hline Run No & Sample Nos. & Type & Heat Treat & Description \\
\hline SLD A981020 & PAW980080 & - & LLLHT002 & $\begin{array}{l}3^{\circ} \mathrm{C} / \mathrm{min} \text { to } 300^{\circ} \mathrm{C} \text {, hold } 2 \mathrm{hrs} \text {, heat at } 5^{\circ} \mathrm{C} / \mathrm{min} \\
\text { to } 1350^{\circ} \mathrm{C} \text {, hold for } 4 \mathrm{hrs} \text {, cool at } 5^{\circ} \mathrm{C} / \mathrm{min} \text { to } \mathrm{RT}\end{array}$ \\
\hline SLD A981021 & LLL153 & I & LLLHT002 & $\begin{array}{l}3^{\circ} \mathrm{C} / \mathrm{min} \text { to } 300^{\circ} \mathrm{C} \text {, hold } 2 \mathrm{hrs} \text {, heat at } 5^{\circ} \mathrm{C} / \mathrm{min} \\
\text { to } 1350^{\circ} \mathrm{C} \text {, hold for } 4 \mathrm{hrs} \text {, cool at } 5^{\circ} \mathrm{C} / \mathrm{min} \text { to } \mathrm{RT}\end{array}$ \\
\hline SLD A981022 & LLL242 & III & LLLHT002 & $\begin{array}{l}3^{\circ} \mathrm{C} / \mathrm{min} \text { to } 300^{\circ} \mathrm{C} \text {, hold } 2 \mathrm{hrs} \text {, heat at } 5^{\circ} \mathrm{C} / \mathrm{min} \\
\text { to } 1350^{\circ} \mathrm{C} \text {, hold for } 4 \mathrm{hrs} \text {, cool at } 5^{\circ} \mathrm{C} / \text { min to } \mathrm{RT}\end{array}$ \\
\hline SLD A981025 & LLL233 & I & LLLHTO02 & $\begin{array}{l}3^{\circ} \mathrm{C} / \mathrm{min} \text { to } 300^{\circ} \mathrm{C} \text {, hold } 2 \mathrm{hrs} \text {, heat at } 5^{\circ} \mathrm{C} / \mathrm{min} \\
\text { to } 1350^{\circ} \mathrm{C} \text {, hold for } 4 \mathrm{hrs} \text {, cool at } 5^{\circ} \mathrm{C} / \mathrm{min} \text { to } \mathrm{RT}\end{array}$ \\
\hline SLD A981027 & $\begin{array}{l}\text { LLL234 } \\
\text { LLL244 }\end{array}$ & I & LLLHT001 & $\begin{array}{l}3^{\circ} \mathrm{C} / \mathrm{min} \text { to } 300^{\circ} \mathrm{C} \text {, hold } 2 \mathrm{hrs} \text {, heat at } 2.5^{\circ} \mathrm{C} / \mathrm{min} \\
\text { to } 1350^{\circ} \mathrm{C} \text {, hold for } 4 \mathrm{hrs} \text {, cool at } 5^{\circ} \mathrm{C} / \mathrm{min} \text { to } \mathrm{RT}\end{array}$ \\
\hline SLD A981029 & $\begin{array}{l}\text { LLL235 } \\
\text { LLL245 }\end{array}$ & $\begin{array}{l}\text { I } \\
\text { III }\end{array}$ & LLLHT005 & $\begin{array}{l}1^{\circ} \mathrm{C} / \mathrm{min} \text { to } 300^{\circ} \mathrm{C} \text {, hold } 2 \mathrm{hrs} \\
\text { cool at } 5^{\circ} \mathrm{C} / \mathrm{min} \text { to } \mathrm{RT}\end{array}$ \\
\hline SLD A981030 & $\begin{array}{l}\text { LLL236 } \\
\text { LLL246 }\end{array}$ & I & LLLHT007 & $\begin{array}{l}0.5^{\circ} \mathrm{C} / \mathrm{min} \text { to } 300^{\circ} \mathrm{C} \text {, hold } 2 \mathrm{hrs} \\
\text { cool at } 5^{\circ} \mathrm{C} / \mathrm{min} \text { to } \mathrm{RT}\end{array}$ \\
\hline SLD A981214 & LLL240 & II & LLLHTO04 & $\begin{array}{l}3^{\circ} \mathrm{C} / \mathrm{min} \text { to } 300^{\circ} \mathrm{C} \text {, hold } 1 \mathrm{hrs}, \\
\text { cool at } 5^{\circ} \mathrm{C} / \mathrm{min} \text { to } \mathrm{RT}\end{array}$ \\
\hline SLD A981215 & LLL162 & II & LLLHTO02 & $\begin{array}{l}3^{\circ} \mathrm{C} / \mathrm{min} \text { to } 300^{\circ} \mathrm{C} \text {, hold } 2 \mathrm{hrs} \text {, heat at } 5^{\circ} \mathrm{C} / \mathrm{min} \\
\text { to } 1350^{\circ} \mathrm{C} \text {, hold for } 4 \mathrm{hrs} \text {, cool at } 5^{\circ} \mathrm{C} / \mathrm{min} \text { to } \mathrm{RT}\end{array}$ \\
\hline SLD A981217 & $\begin{array}{l}\text { LLL237 } \\
\text { LLL167 }\end{array}$ & I & LLLHT008 & $\begin{array}{l}3^{\circ} \mathrm{C} / \mathrm{min} \text { to } 110^{\circ} \mathrm{C} \text {, hold } 1 \mathrm{hr}, 3^{\circ} \mathrm{C} / \mathrm{min} \text { to } \\
300^{\circ} \mathrm{C} \text {, hold } 2 \mathrm{hrs} \text {, heat at } 5^{\circ} \mathrm{C} / \mathrm{min} \text { to } 1350^{\circ} \mathrm{C} \text {, } \\
\text { hold for } 4 \mathrm{hrs} \text {, cool at } 5^{\circ} \mathrm{C} / \mathrm{min} \text { to } \mathrm{RT}\end{array}$ \\
\hline SLD A981222 & $\begin{array}{l}\text { LLL239CIP } \\
\text { LLL168CIP }\end{array}$ & II & LLLHT008 & $\begin{array}{l}3^{\circ} \mathrm{C} / \mathrm{min} \text { to } 110^{\circ} \mathrm{C} \text {, hold } 1 \mathrm{hr}, 3^{\circ} \mathrm{C} / \mathrm{min} \text { to } \\
300^{\circ} \mathrm{C} \text {, hold } 2 \mathrm{hrs} \text {, heat at } 5^{\circ} \mathrm{C} / \mathrm{min} \text { to } 1350^{\circ} \mathrm{C} \text {, } \\
\text { hold for } 4 \mathrm{hrs} \text {, cool at } 5^{\circ} \mathrm{C} / \mathrm{min} \text { to } \mathrm{RT}\end{array}$ \\
\hline
\end{tabular}

Dr. Philip Walls ANSTO Materials Division 
Table 4. Summary of Sample Data.

\begin{tabular}{|c|c|c|c|c|c|c|c|c|c|c|c|}
\hline \multirow{2}{*}{\multicolumn{2}{|c|}{$\begin{array}{r}\text { Type } \\
\text { Orgin Isamplewo: }\end{array}$}} & 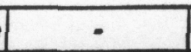 & 7 & III & 1 & 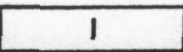 & III & 1 & III & 8 & III \\
\hline & & SYNBUN/43 & 463 & 4242 & 1921238 & LLIL234 & MII 244 & 42335 & 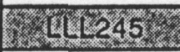 & 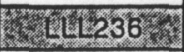 & 24,64 \\
\hline \multicolumn{2}{|c|}{ Sample Register No. } & PAW980080 & PAW980081 & PAW980082 & PAW980083 & PAW980084 & PAW980085 & PAW980086 & PAW980087 & PAW980088 & PAW980089 \\
\hline 8 & SED & A 48.620 & 349187024 & 4089025 & 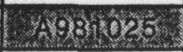 & 198102 & 4981027 & 4081029 & . & Kgraso & 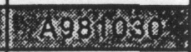 \\
\hline \multicolumn{2}{|c|}{ Heat Treatment No. } & SYNHTOO2 & LLLHT002 & LLLLTT002 & LLLHTO02 & LLLLHT001 & LLLHT001 & LLLHTT005 & LLLHT005 & LLLHTO07 & LLLHTO07 \\
\hline \multirow{4}{*}{$\begin{array}{l}\text { Weight } \\
\text { (g) }\end{array}$} & Iniltial & 4520.0 & 8745000 & 4535 & 4333.5 & 324.0 & 465.0 & 324.5 & 445.0 & 327.0 & 447.0 \\
\hline & Final & $20513.5 \mathrm{~kg}$ & $84240 \%$ & 39970 & 81807.0717 & 296.0 & $6-408: 5$ & 316.4 & 423.5 & 317.5 & 428.5 \\
\hline & Difference & 66.5 & $260 \%$ & 1056.5 & 826.5 & 28.0 & 56.5 & 8.1 & 21.5 & 9.5 & 5 \\
\hline & $\%$ Diff & $80^{-1.3}, \mathrm{k}$ & 36285.812 & 2125 & 807.9 & 8.6 & 12.2 & 2.5 & 4.8 & 2.9 & 4.1 \\
\hline \multirow{4}{*}{$\begin{array}{l}\text { otamater } \\
\text { (min) }\end{array}$} & Finfital & 89.99 & 89.85 & 8998 & 8920 & 89177 & 89.12 & 89.172 & C.89611 & $89: 18$ & 896 \\
\hline & Enoth & $\sqrt{7} 24 \times 8$ & 66569 & 70060 & 6576 & 65501 & 20,172 & 489212 & 8920 & 8915 & 39983 \\
\hline & pliference: & 48015 & 28660 & 18068 & 28844 & 231678 & 1895 & 80004 & 200969 & 0003 & rot2 \\
\hline & \% Diff & 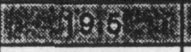 & 36.6 & 2009 & 42668 & 2675 & 21,3 & 800002 & $3 \times 140016$ & 000 & 4022 \\
\hline \multirow{4}{*}{$\begin{array}{l}\text { Thickness } \\
\text { (mm) }\end{array}$} & Initial & 40.76 & 35.45 & 28.67 & 25.70 & 24.88 & 29.55 & 25.07 & 28. & 25.40 & 29.42 \\
\hline & Final & 30.85 & 25.92 & 2274 & 18.80 & 18.12 & 7 & 25.22 & 28.62 & 25.30 & 9.3 \\
\hline & Difference & 9.91 & 9.53 & 5.93 & 6.90 & 6.76 & 6.18 & -0.15 & -0. & 0.10 & .0 \\
\hline & $\%$ Diff & 24.3 & 26.9 & 20,7 & 26.8 & 27.2 & 20. & -0.6 & -0. & 0.4 & $0.2^{2}+x-1$ \\
\hline \multirow{4}{*}{$\begin{array}{l}\text { Volume } \\
\text { (cc) } \\
\text { of }\end{array}$} & Initial & 259.1 & 2212 & 9990 & $160: 5$ & 155.3 & 184.2 & 156.5 & 177.4 & 158.6 & $183: 4$ \\
\hline & Final & 9127.2 & 384 & 888 & $63: 8$ & 61.0 & 90.3 & 157.6 & 178 & 157.8 & 183.8 \\
\hline & Difference & 131192 & 63 & 909 & 967 & 94.3 & 93.9 & 201.1 & $8-1.4$ & 5.0 .716 & $89:-0.4$ \\
\hline & $\%$ Diff $3 \%$ & 850.9 & 6005 & 5094 & 6022 & 60.7 & 51.0 & -0.7 & -0.8 & 0.5 & -0.2 \\
\hline oponing & 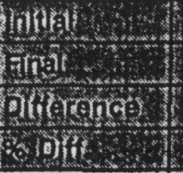 & $\frac{804}{904}$ & 09 & 53 & 5.83 & 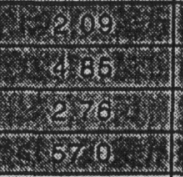 & $\frac{2625}{26009}$ & $\frac{\frac{307}{3075}}{\frac{0.07}{3.6}}$ & 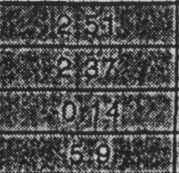 & $\frac{\frac{206}{307}}{\frac{3005}{25}}$ & 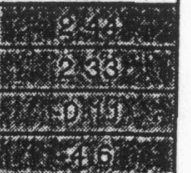 \\
\hline 10 & $(8 / \mathrm{cc})$ & 4.4508 & 620 & 520 & 520 & 5.20 & 5752007 & 5520512 & 515,200 & 885.2017 & 55208 \\
\hline & & & & & & & & & & & \\
\hline
\end{tabular}

NB. TD of $5.2 \mathrm{~g} / \mathrm{cc}$ is approximate for Ce baseline composition 


\begin{tabular}{|c|c|c|c|c|c|c|c|}
\hline \multicolumn{2}{|c|}{ Type } & $\pi$ & II & 1 & IIII & $\| \mathrm{CIPed}$ & III ClPed \\
\hline \multirow{2}{*}{\multicolumn{2}{|c|}{ Sample Register No. }} & 0,45640 & 41462 & YLEL237 & 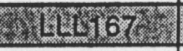 & PLLL239: & YLLL168: \\
\hline & & PAW980090 & PAW980091 & PAW980092 & PAW980093 & PAW980094 & PAW980095 \\
\hline \multicolumn{2}{|c|}{ 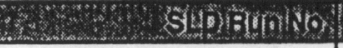 } & 6081212 & 149812156 & 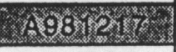 & 48813217 & 18961252 & 舞 \\
\hline \multicolumn{2}{|c|}{ Heat Treatment No. } & LLLHTO05 & LLLLTT002 & LLLHTO08 & LLLHTO08 & LLLHTO08 & LLLHT008 \\
\hline \multirow{8}{*}{$\begin{array}{l}\text { Weight } \\
\text { (g) }\end{array}$} & Inittal: & & 398.0 & 322.0 & 562.5 & 457.5 & 608.5 \\
\hline & Finalses & 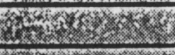 & 31368.5 & 292.5 & 494.5 & 422.0 & 536.0 \\
\hline & Differentice & mex & 29.5 & 29.5 & 68.0 & $\begin{array}{r}35.5 \\
\end{array}$ & 72.5 \\
\hline & \%opiff rax & \% & 27.41 & 9.2 & 12.1 & 7.8 & 11.9 \\
\hline & inital & & $896 \pi 6$ & 89612 & 9804482 & 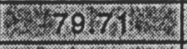 & 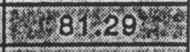 \\
\hline & Engal & & 668783 & 65538 & 68927 & 2642967 & $6868: 50 \% 4$ \\
\hline & Difforeicos & & 25338 & 2379 & 20.22 & $15: 42$ & 12.79 \\
\hline & 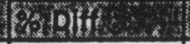 & & 28.4 & $262 \%$ & 227 & $201918=2$ & $15 \% 7$ \\
\hline \multirow{4}{*}{$\begin{array}{l}\text { Thickness } \\
(\mathrm{mm})\end{array}$} & Initial & & 29.68 & 25.20 & 37.90 & 33.32 & 37.22 \\
\hline & Final & & 23.73 & 18.90 & 30.11 & 26.73 & 30.66 \\
\hline & Difference & & 5.95 & 6.30 & 7.79 & 6.59 & 6.56 \\
\hline & $\%$ Diff & & 20.0 & 25.0 & 20.6 & 19.8 & 17.6 \\
\hline \multirow{4}{*}{$\begin{array}{l}\text { Volume } \\
\text { (cc) }\end{array}$} & Initialy & & 185.22 & $2821=0.0$ & semetery & 166.2 & 193.1 \\
\hline & Enall & & 55.9 & 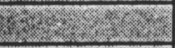 & 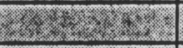 & 86.7 & 112.9 \\
\hline & Ditterence & 68 & 700.3 & 32.20 & $278 \div 28$ & 79.5 & \begin{tabular}{|l}
80.1 \\
\end{tabular} \\
\hline & \%olf & 2 & $2859: 0$ & $28 x^{25}=2$ & 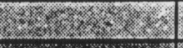 & 47.8 & 41.5 \\
\hline \multirow{3}{*}{ 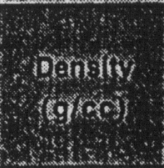 } & Initgen & & 28 & 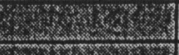 & & 5 & 3.15\% \\
\hline & Fial & & 488 & 48 & 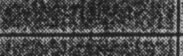 & 4 & $5 \%$ \\
\hline & bifformose & & & $\frac{1120}{2 \times 3}$ & 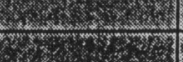 & $\frac{6227 \pi}{34}$ & 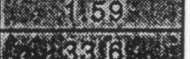 \\
\hline \multirow{3}{*}{$\frac{\pi}{\mathrm{T} D}$} & (cibor & & $520 \%$ & 65.20 & 6851205 & $560 \%$ & 16: \\
\hline & $2 \%$ & 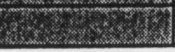 & $93.4 \times$ & 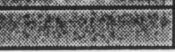 & 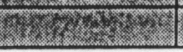 & 93.66 & $91: 3$ \\
\hline & & & & & & & \\
\hline
\end{tabular}




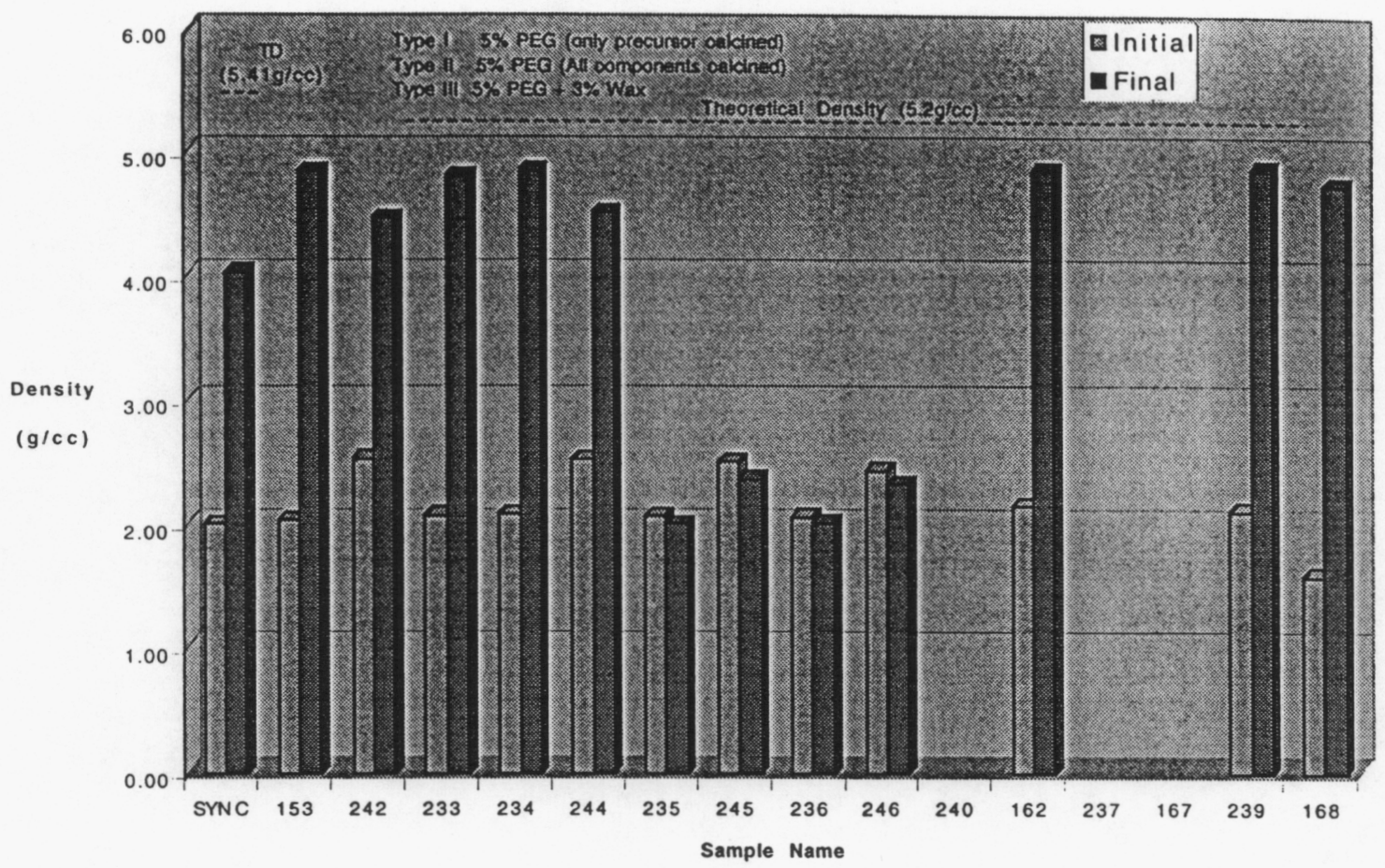

Figure 1 Initial \& Final Densities

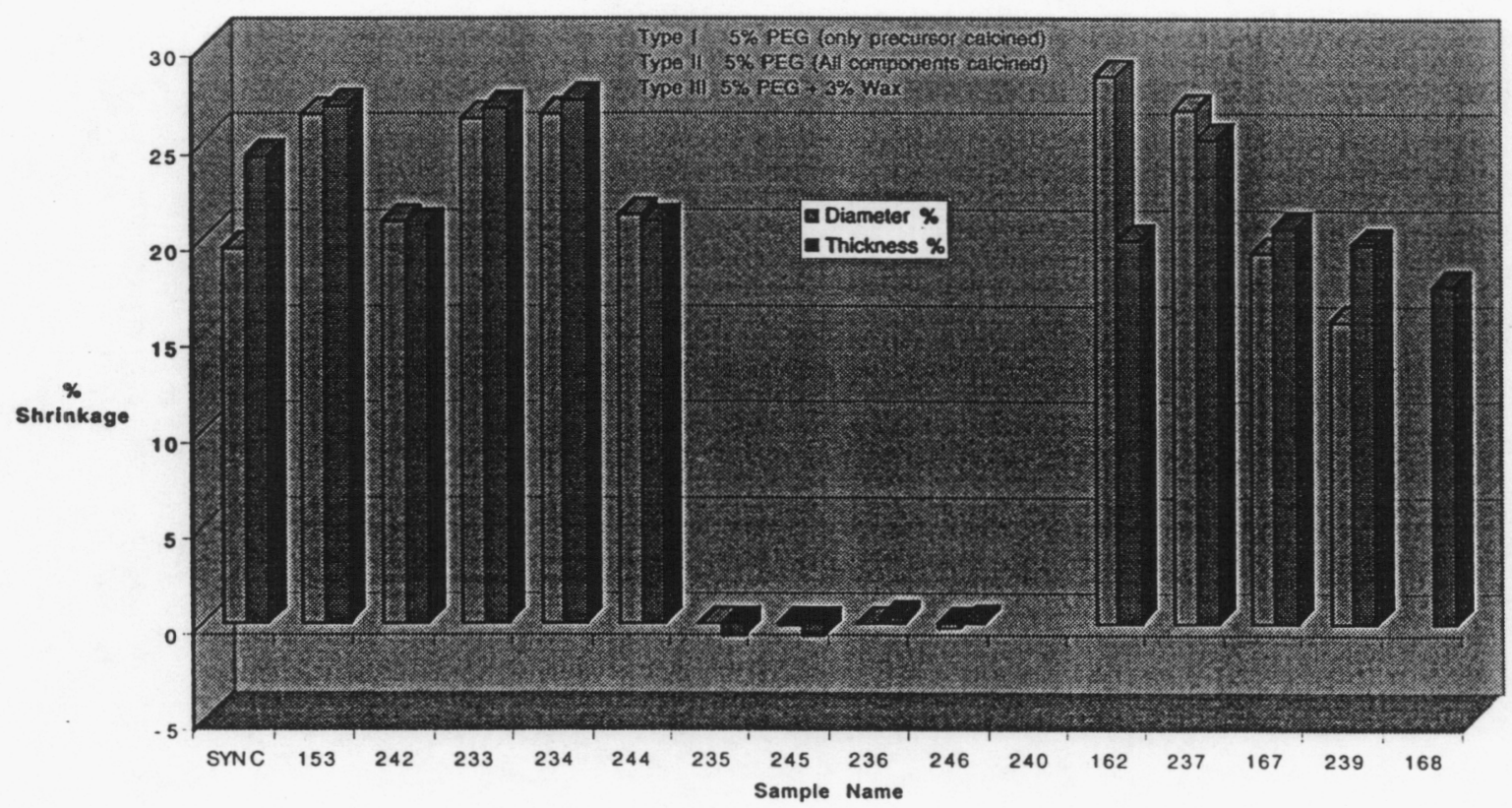

Figure 2 Sintering Shrinkage 


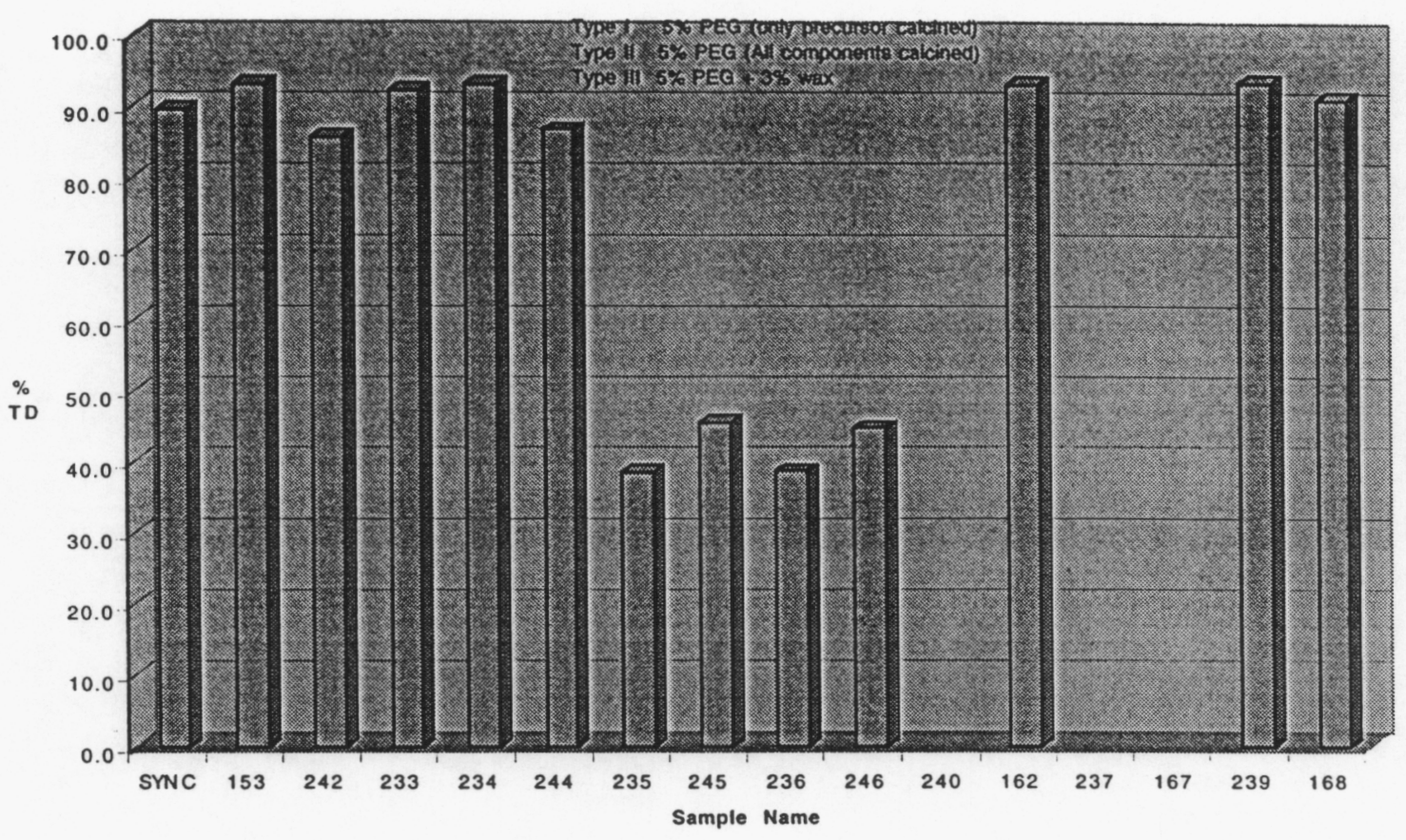

Figure $3 \%$ Theoretical Density

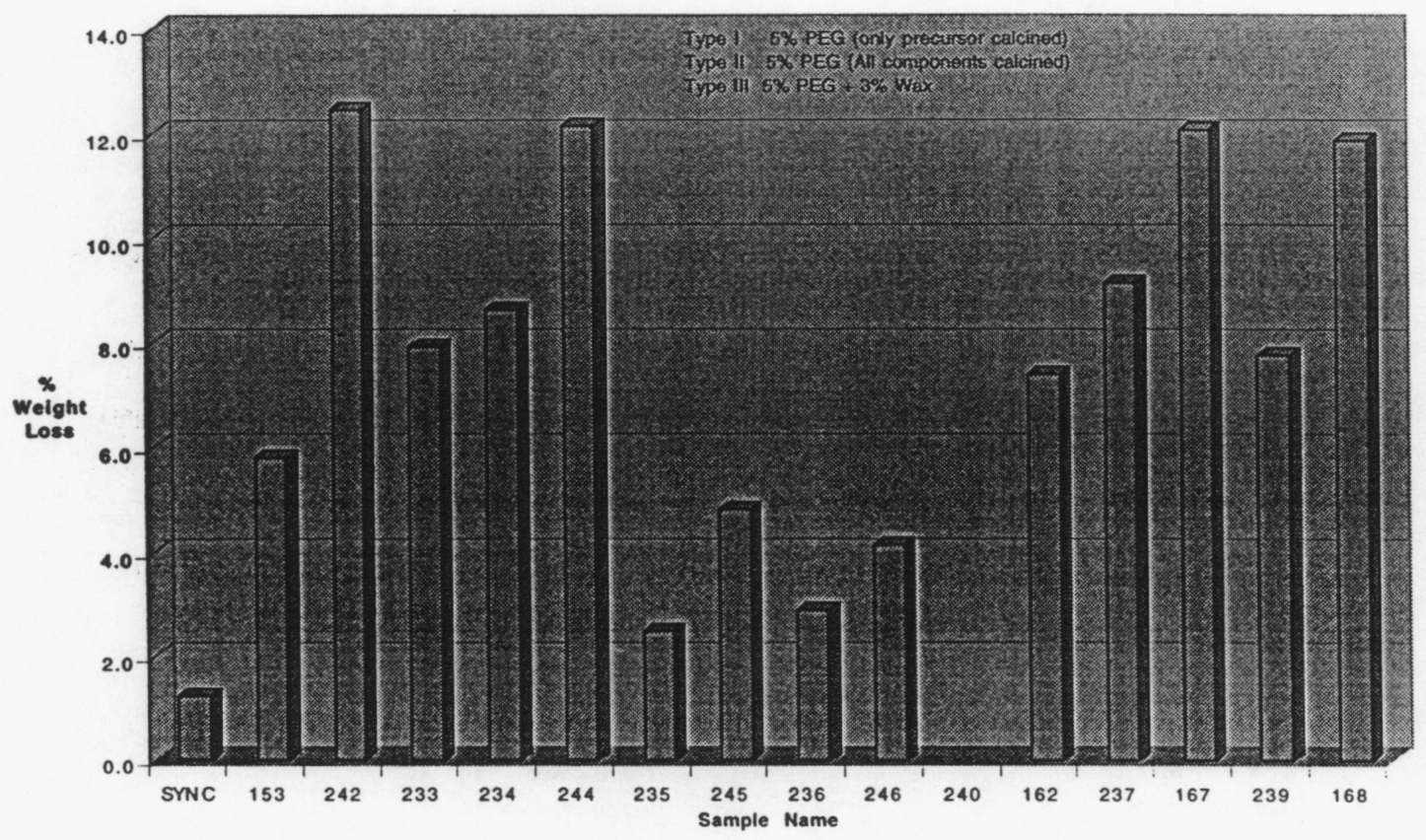

Figure 4 Weight Loss from Samples 


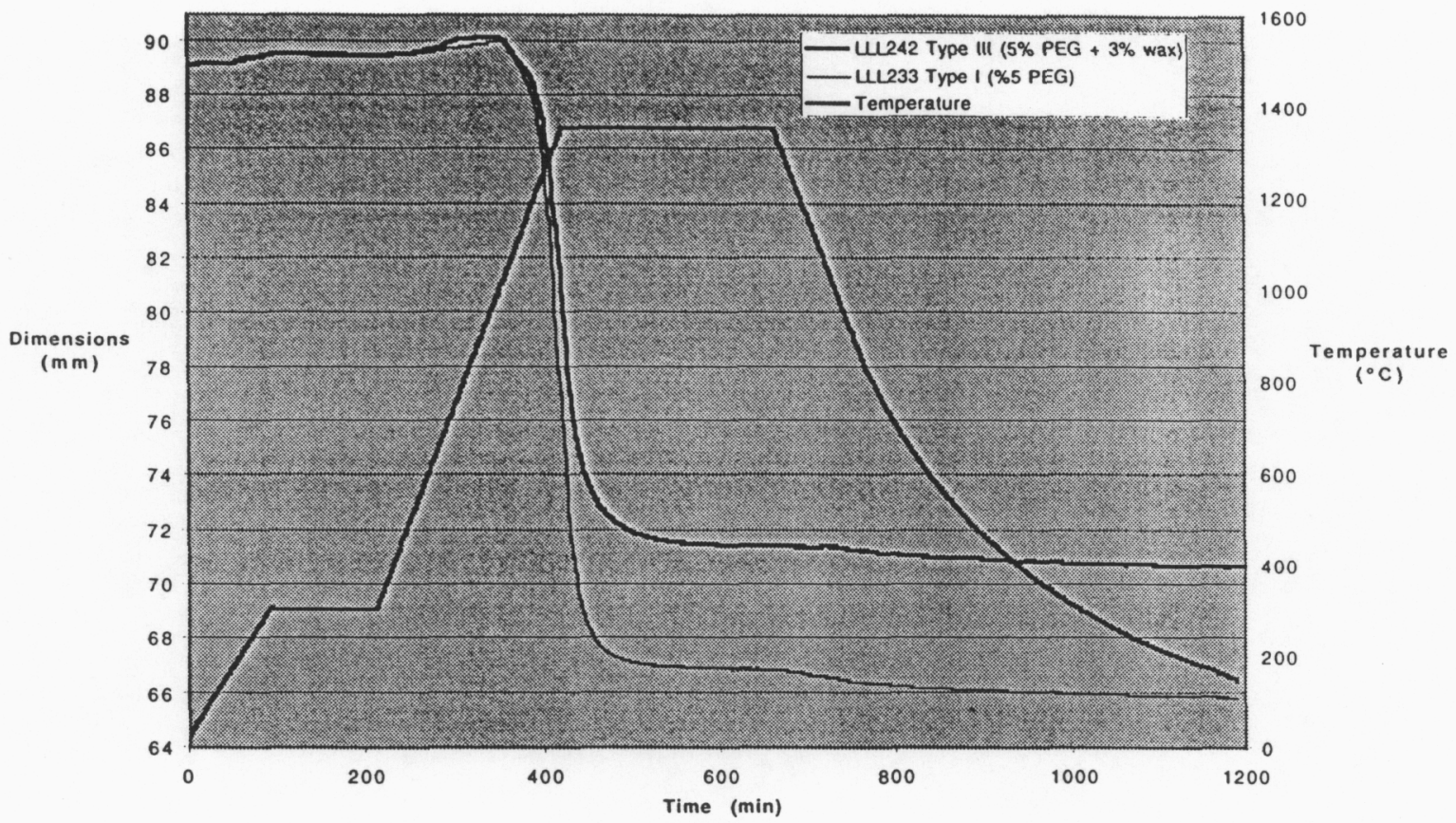

Figure 5 SLD Runs A981020 \& A981021

Table 6. Moisture Content of Samples

\begin{tabular}{|c|c|c|c|}
\hline Sample No. & \%PEG & \%wax & \%wt loss \\
\hline Synroc C (Run 143) & 0 & 0 & 0.29 \\
\hline LLL233 (Type I) & 5 & 0 & 0.89 \\
\hline LLL243 (Type III) & 5 & 3 & 1.52 \\
\hline
\end{tabular}

Run SLD A981025 : Run using standard baseline heat treatment. LLL233 (Type I) sample containing $5 \%$ PEG, which had been dried at $50^{\circ} \mathrm{C}$ for $20 \mathrm{hrs}$. The sample cracked in a more severe manner than that in the previous run, which indicated that moisture content was not the most serious factor in maintaining the integrity of the sample, see Figure 19.

The low temperature region $\left(20^{\circ} \mathrm{C}\right.$ to $\left.400^{\circ} \mathrm{C}\right)$ of the dilatometer data was then investigated in detail. It was found that between $170^{\circ} \mathrm{C}$ and $300^{\circ} \mathrm{C}$, both Type I and III samples dilated more rapidly compared to heating between $20^{\circ} \mathrm{C}$ to $170^{\circ} \mathrm{C}$. This phenomenon is shown in Figure 11 with a Synroc C (Run 143) sample for comparison.

The CIPed, zero organics, Synroc C sample expanded uniformly till $300^{\circ} \mathrm{C}$, stayed at a constant diameter during the $120 \mathrm{~min}$ hold, then continued expanding once the temperature began to ramp up to $1350^{\circ} \mathrm{C}$. Both the Type I and Type III samples expanded 
uniformly until $170^{\circ} \mathrm{C}$, the rate of expansion increased between $170^{\circ} \mathrm{C}$ and $300^{\circ} \mathrm{C}$, and then there was a contraction of the sample during the hold. It is proposed that this departure from the observed linear expansion of the sample represents the initiation and growth of cracks within the body of the sample. NB. A number of ANSTO staff were called to witness that the samples had indeed cracked by the time the sample had reached $300^{\circ} \mathrm{C}$.

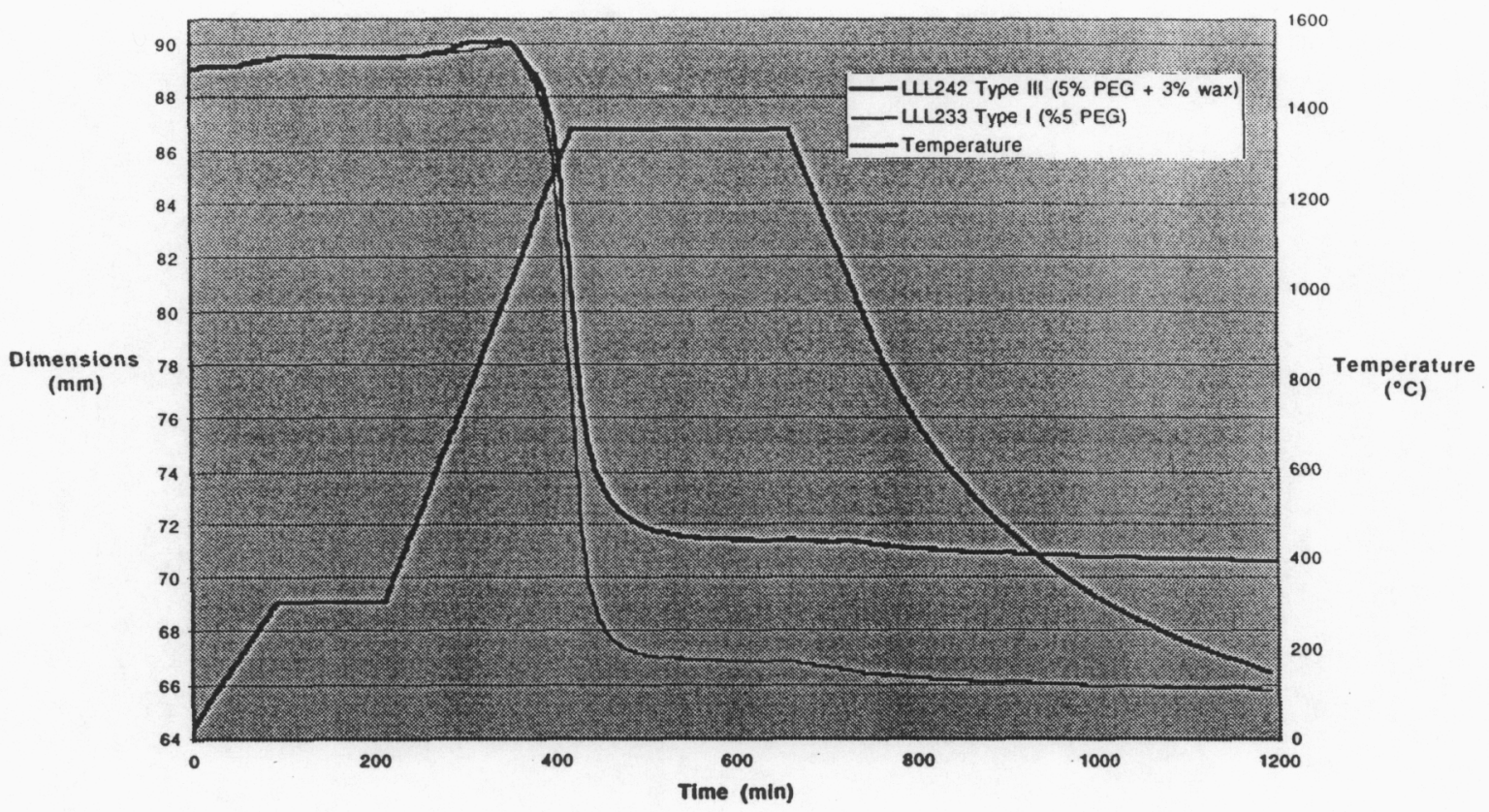

\section{Figure 6. SLD Run A981022 \& SLD Run A981025 $3^{\circ} \mathrm{C} / \mathrm{min}$ to $300^{\circ} \mathrm{C}$ $5^{\circ} \mathrm{C} / \mathrm{min}$ to $1350^{\circ} \mathrm{C}$ with a $4 \mathrm{hr}$ hold}

Run SLD A981027 : In order to prove that the cracks were not the result of rapid temperature ramping between $300^{\circ} \mathrm{C}$ and $1350^{\circ} \mathrm{C}$. This run was performed at $2.5^{\circ} \mathrm{C} / \mathrm{min}$ between $300^{\circ} \mathrm{C}$ and $1350^{\circ} \mathrm{C}$, cf. $5^{\circ} \mathrm{C} / \mathrm{min}$ in the previous run. Two samples (LLL234 Type I \& LLL244 Type III) were simultaneously monitored in this run. There was no significant difference in the cracking observed in this run at $2.5^{\circ} \mathrm{C} / \mathrm{min}$ heating rate, $\mathrm{cf}$. $5^{\circ} \mathrm{C} / \mathrm{min}$ in Run SLD A981025, see Figures 13 \& 14. 


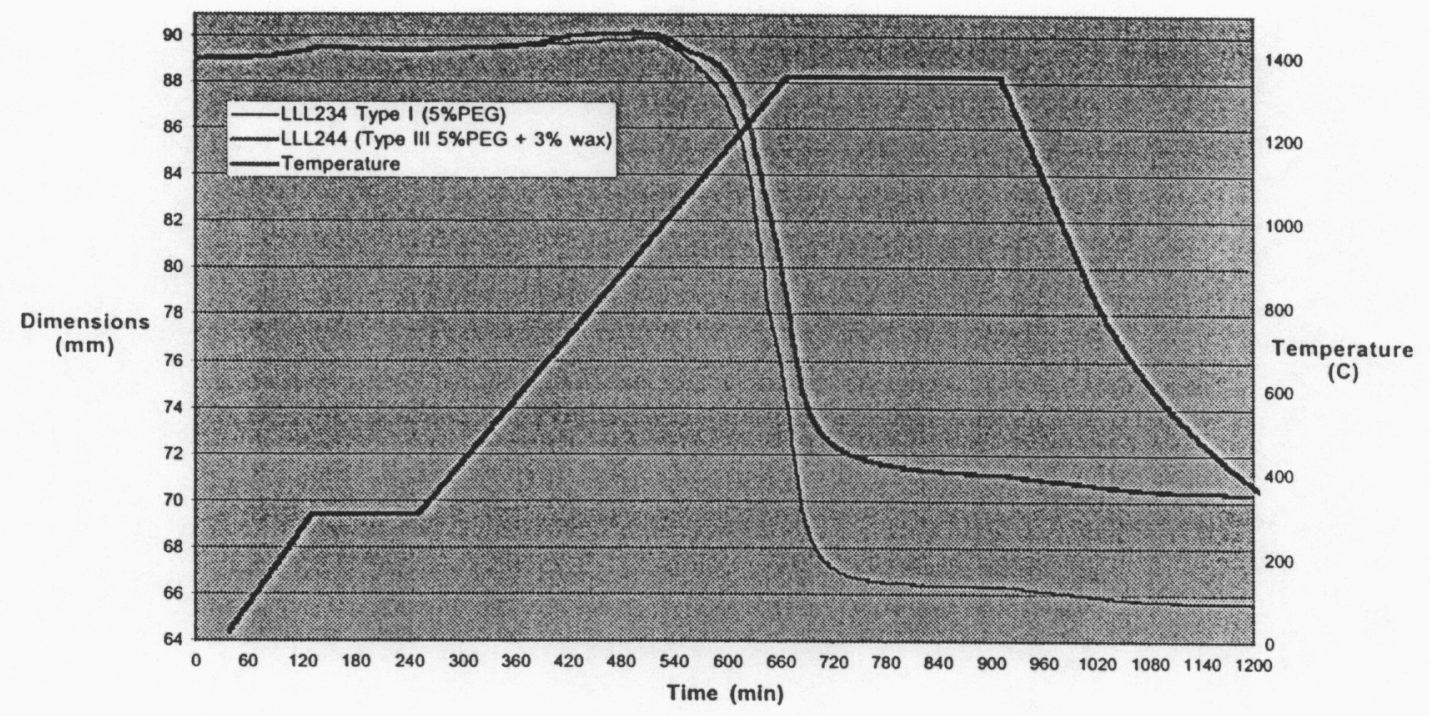

Figure 7. SLD Run A981027 $3^{\circ} \mathrm{C} / \mathrm{min}$ to $300^{\circ} \mathrm{C} 2.5^{\circ} \mathrm{C} / \mathrm{min}$ to $1350^{\circ} \mathrm{C}$, $4 \mathrm{hr}$ hold

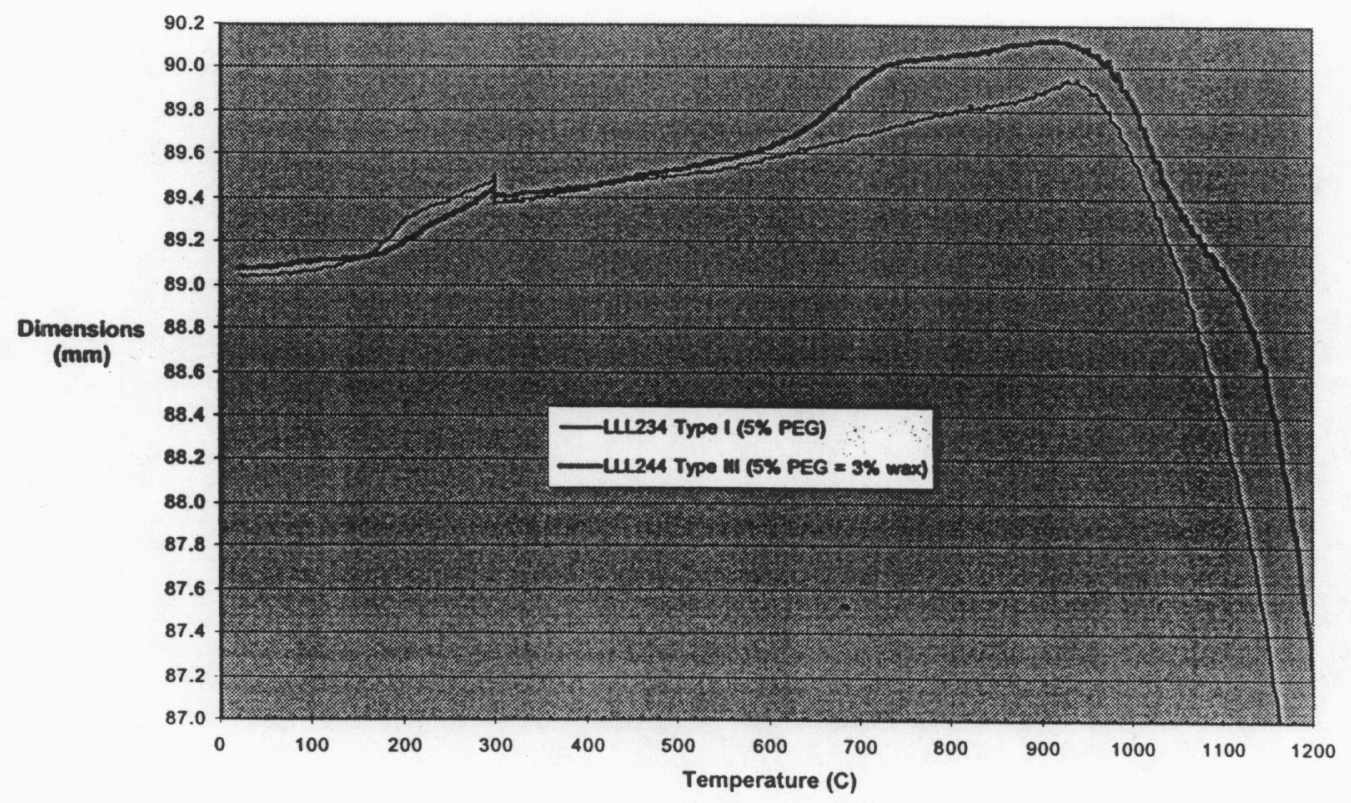

Figure 8. Detail of SLD Run A981027

Run SLD A981029 : This experiment looked at the effect of reducing the heating rate between room temperature (RT) and $300^{\circ} \mathrm{C}$. A rate of $1{ }^{\circ} \mathrm{C} / \mathrm{min}$ was used to examine 
whether the degree of cracking could be alleviated or eliminated altogether. It was decided to cool the sample to RT after the $120 \mathrm{~min}$ hold at $300^{\circ} \mathrm{C}$ to examine the samples, see Figures 15 \& 16. Cracking still occurred, and incomplete burnout of the binder and wax were observed on the underside of the samples, which had been in contact with the Pt sheet. NB. More burnout was observed in the LLL235 (Type I) sample containing 5\%PEG only, cf. sample LLL245 (Type III) which contained 5\%PEG + 3\%wax.

Run SLD A981030 : In this final run a heating rate of $0.5^{\circ} \mathrm{C} / \mathrm{min}$ was used to $300^{\circ} \mathrm{C}$. The results were similar to the last run $\left(1^{\circ} \mathrm{C} / \mathrm{min}\right)$ however slightly less cracking was observed and even more of the binder and wax had burnt out. The Dimension Change / Temperature Profiles of samples heated to $300^{\circ} \mathrm{C}$ at $1^{\circ} \mathrm{C} / \mathrm{min}$ and $0.5^{\circ} \mathrm{C} / \mathrm{min}$ are shown in Figure 22. Similar non-uniform dilation above $150^{\circ} \mathrm{C}$ is observed in all the samples.

Tables 4 \& 5 summarise the test results so far. Sample dimensions, percentage change and bulk density calculations (from dimensions) are included.

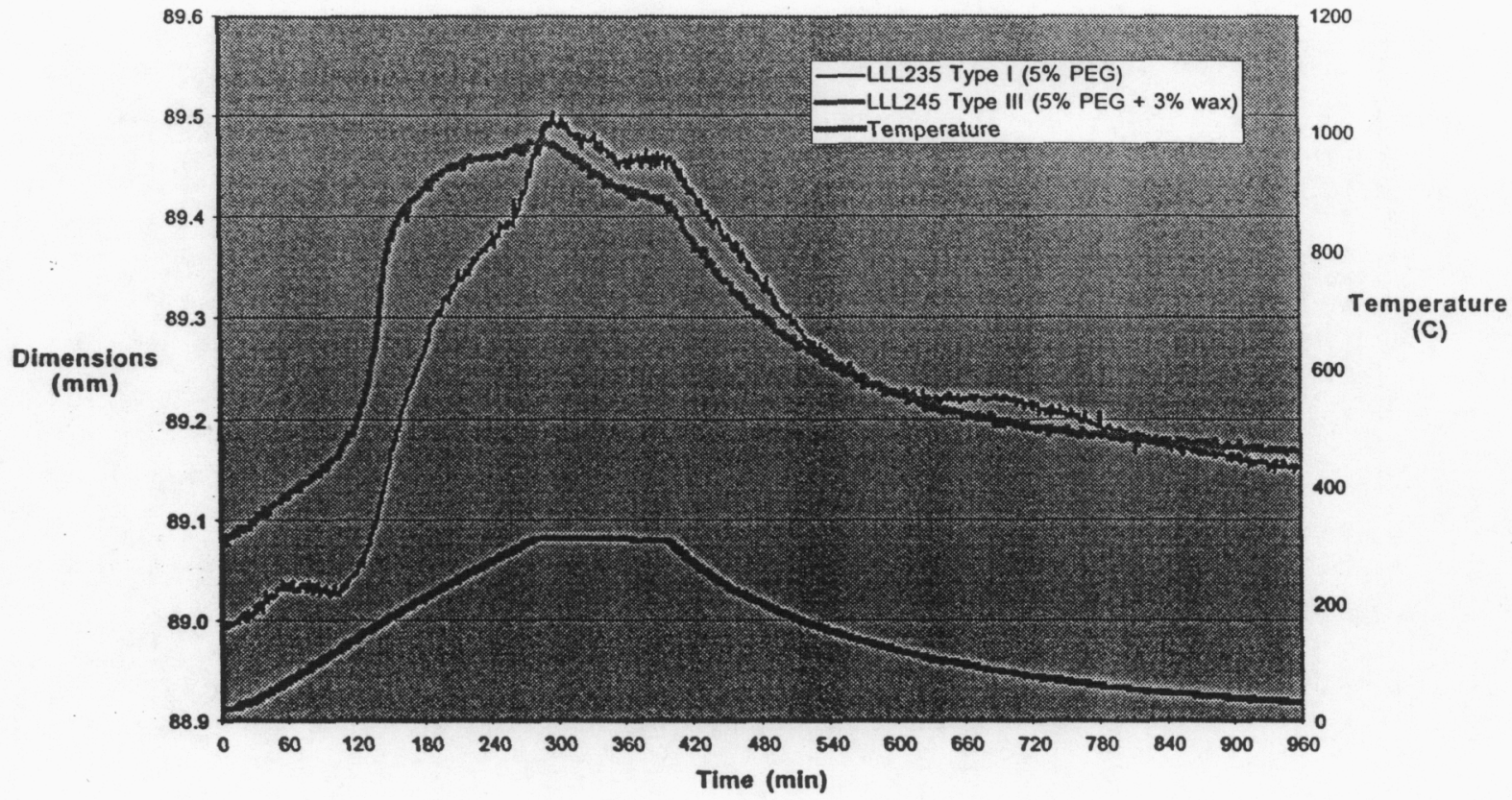

Figure 9. SLD Run A981029 $1^{\circ} \mathrm{C} / \mathrm{min}$ to $300^{\circ} \mathrm{C}$ 


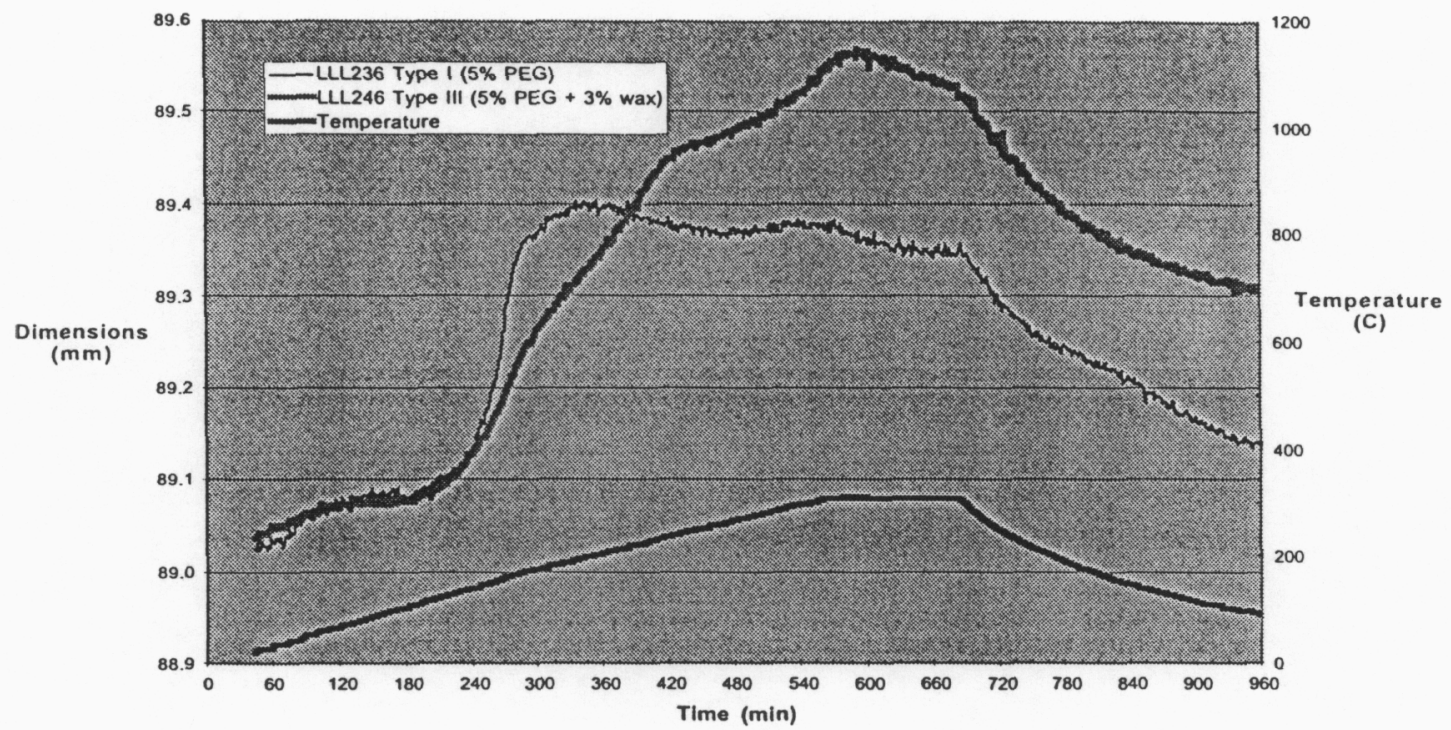

Figure 10. SLD Run A981030 $0.5^{\circ} \mathrm{C} / \mathrm{min}$ to $300^{\circ} \mathrm{C}$

\section{Sample Photographs}

Figures 11 to 21 show optical photographs of the heat-treated samples. Dimensions of these pucks are shown in Table 4. Cracking was observed in all samples, even those heated to $300^{\circ} \mathrm{C}$ at $0.5^{\circ} \mathrm{C} / \mathrm{min}$ (LLL236 \& LLL246). The mode of cracking suggested that the cracks were initiating at low (a few hundred ${ }^{\circ} \mathrm{C}$ ) temperatures, ie. the cracks were quite wide cf. hairline cracks associated with thermal shocking. This was backed up by direct observation of cracks at 200 to $300^{\circ} \mathrm{C}$ by a number of staff through the silica glass windows in the laser dilatometer furnace.

The number and arrangement of cracks were different between the Type I and Type II samples. Typically there were larger, fewer cracks in the Type I (5\%PEG) samples compared too more, finer cracks in the Type III ( $5 \%$ PEG + 3\% wax) samples.

Figures 15 to 18 show the samples which were heated to $300^{\circ} \mathrm{C}$, held at temperature for 120 mins, before being cooled to room temperature at $5^{\circ} \mathrm{C} / \mathrm{min}$. Figures $15 \& 16$ show samples LLL235 and LLL245 which were heated at $1^{\circ} \mathrm{C} / \mathrm{min}$ to $300^{\circ} \mathrm{C}$ (taking $5 \mathrm{hrs)}$ ) and Figures $17 \& 18$ show samples LLL236 and LLL246 which were heated at $0.5^{\circ} \mathrm{C} / \mathrm{min}$ to $300^{\circ} \mathrm{C}$ (taking 10hrs). 

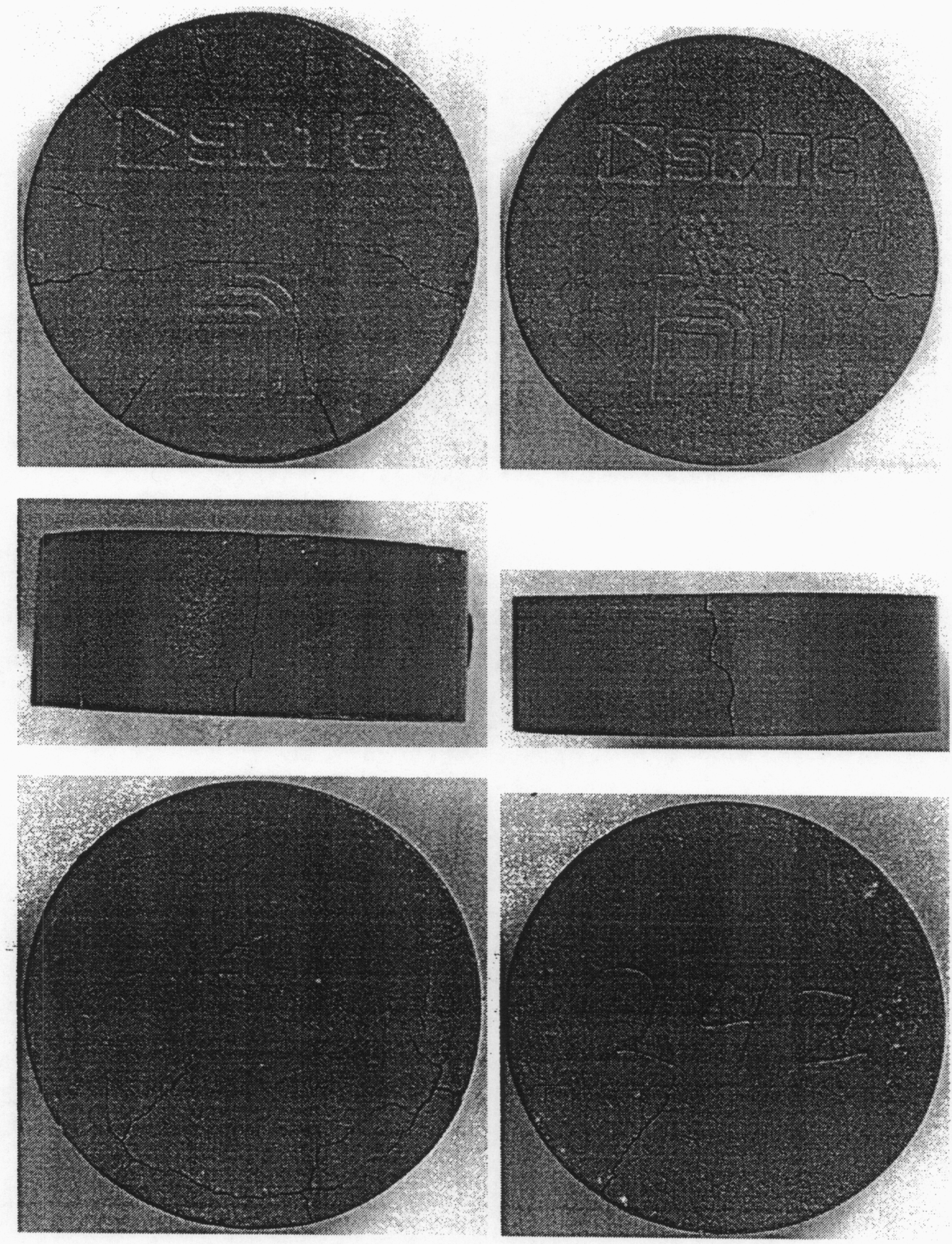

Figure 11 LLL153

Figure 12 LLL153 

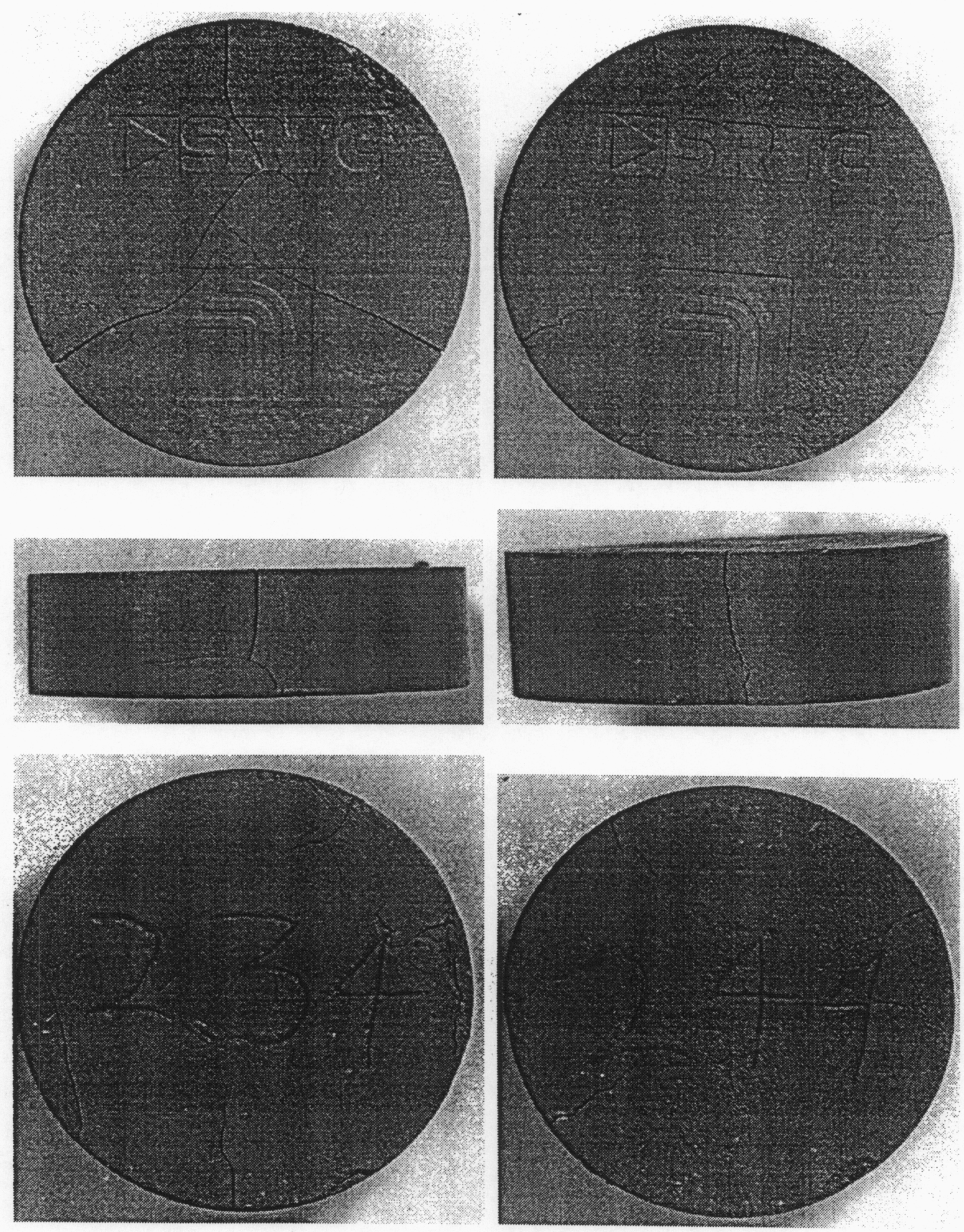

Figure 13 LLL234

Figure 14 LLL244

Dr. Philip Walls ANSTO Materials Division 

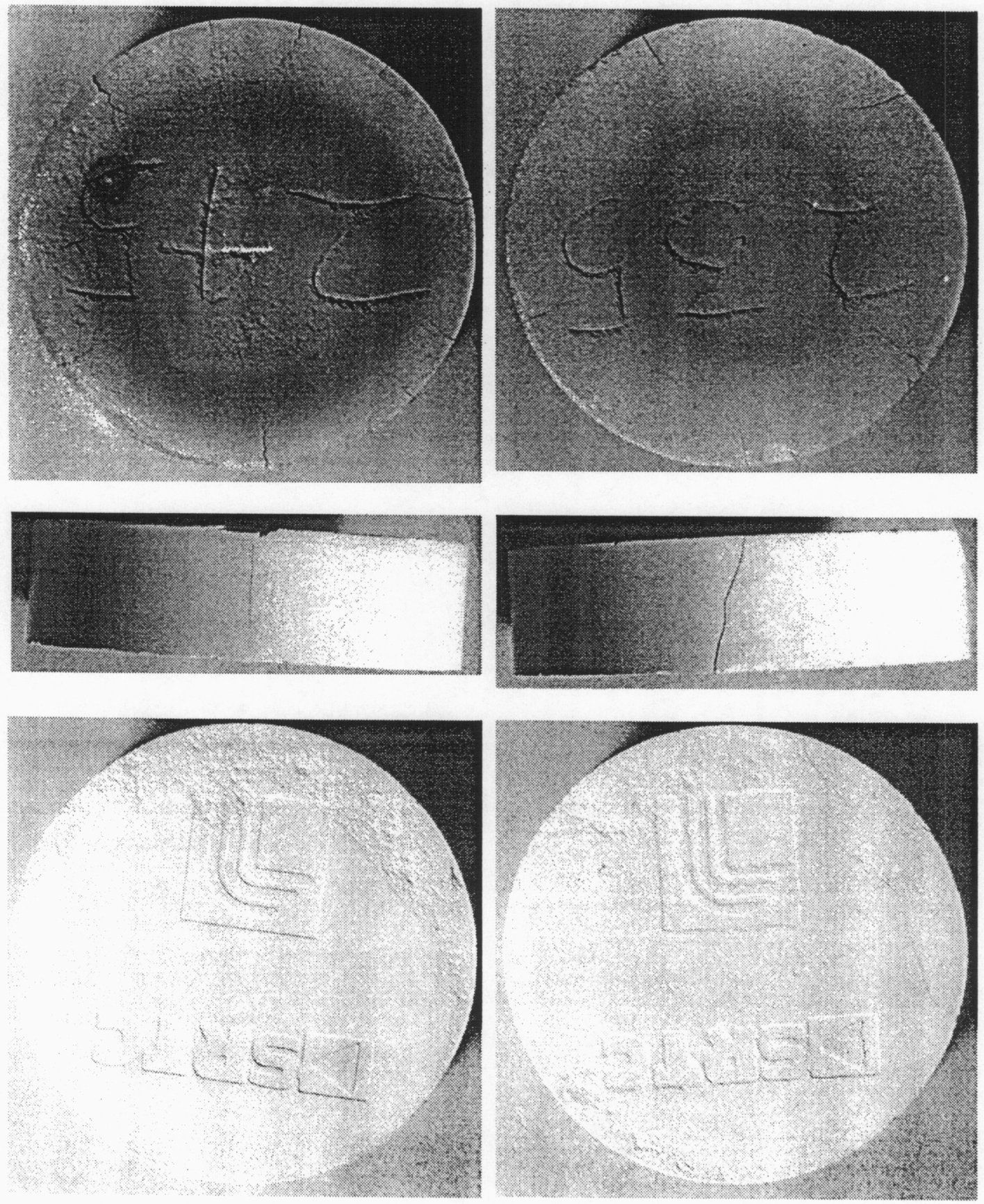

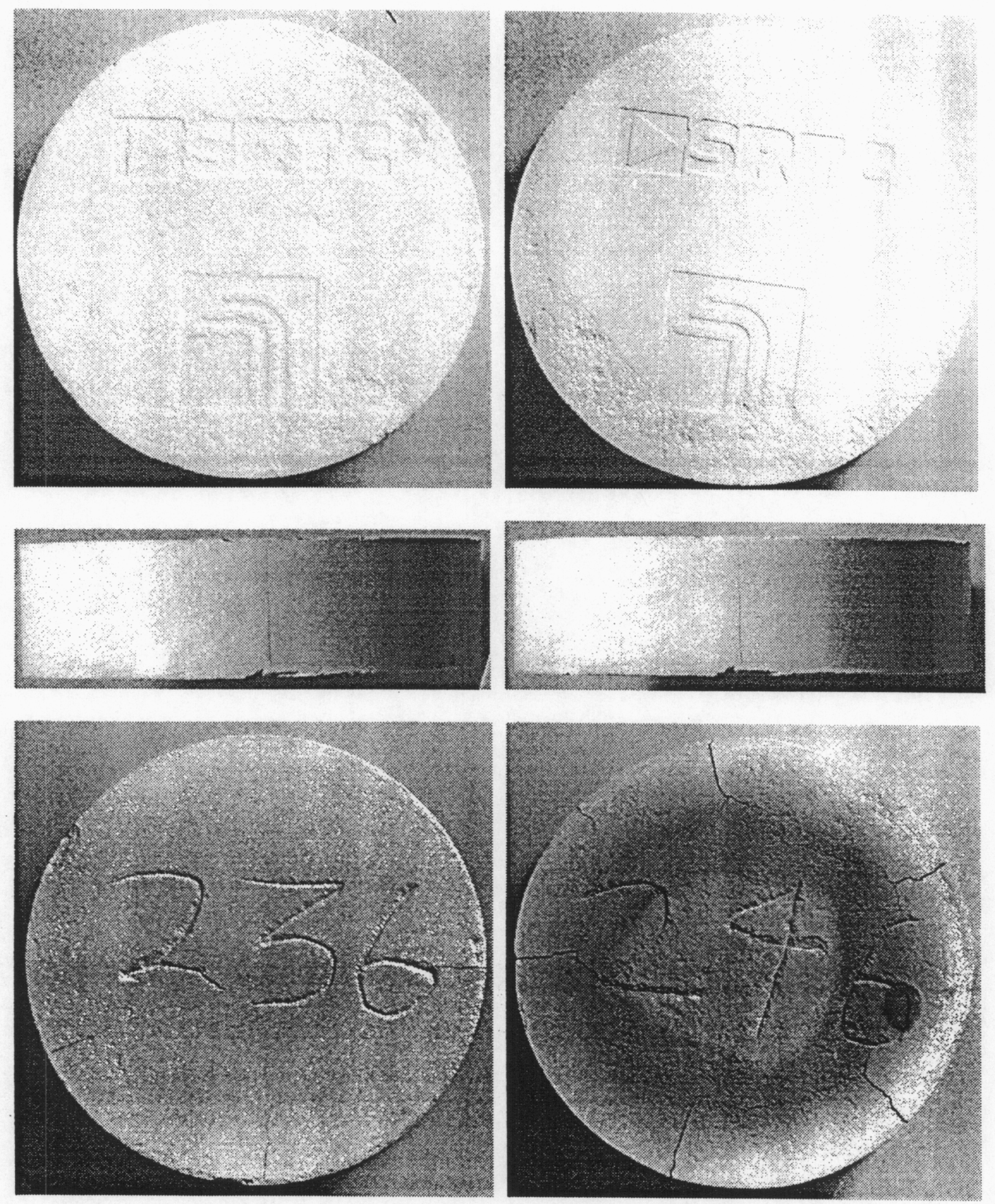

Figure 17 LLL236

Figure 18 LLL246

Dr. Philip Walls ANSTO Materials Division 

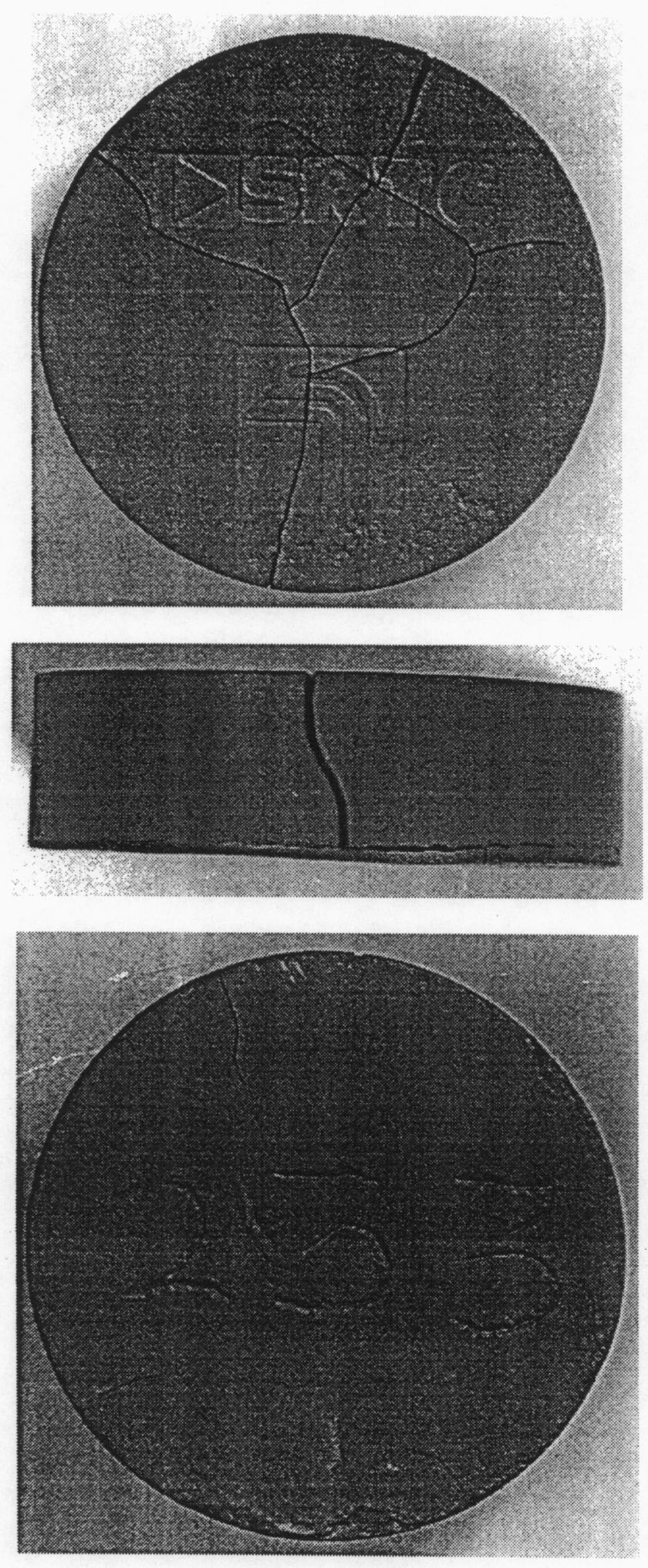

Figure 19 LLL233

Dr. Philip Walls ANSTO Materials Division 


\section{DT/TGA Results}

In order to examine whether volatiles were responsible for the dilation of the samples during heating to $300^{\circ} \mathrm{C}$, small $<0.5 \mathrm{~g}$ pieces of material were broken off the edges of samples LLL235 (Type I) and LLL244 (Type III) for Differential Thermogravimetric Analysis. The spectra obtained for the 2 samples are shown in Figure 20. The LLL235 (Type I) sample contained 5\%PEG only, while LLL244 (Type III) contained $5 \%$ PEG+3\%wax.

The endotherm at $70^{\circ} \mathrm{C}$ is due to the PEG melting. There are large exotherms at $240^{\circ} \mathrm{C}$ and $300^{\circ} \mathrm{C}$, which are attributed to partial decomposition of the PEG and wax respectively. The endotherm at $430^{\circ} \mathrm{C}$ and associated weight loss could be attributed to the boiling off of PEG 8000 . The final weight loss between $600^{\circ} \mathrm{C}$ and $800^{\circ} \mathrm{C}$ is most probably associated with breakdown and boil off of the high MW PEG fractions.

These results show that the PEG and wax are still escaping from the samples at temperatures in excess of $300^{\circ} \mathrm{C}$. It is also interesting that the observed increase in the rate of dilation of the sample between $170^{\circ} \mathrm{C}$ and $300^{\circ} \mathrm{C}$ occur at the same time as the initial decomposition in the 5\% PEG and 5\% PEG+3\% wax containing samples.

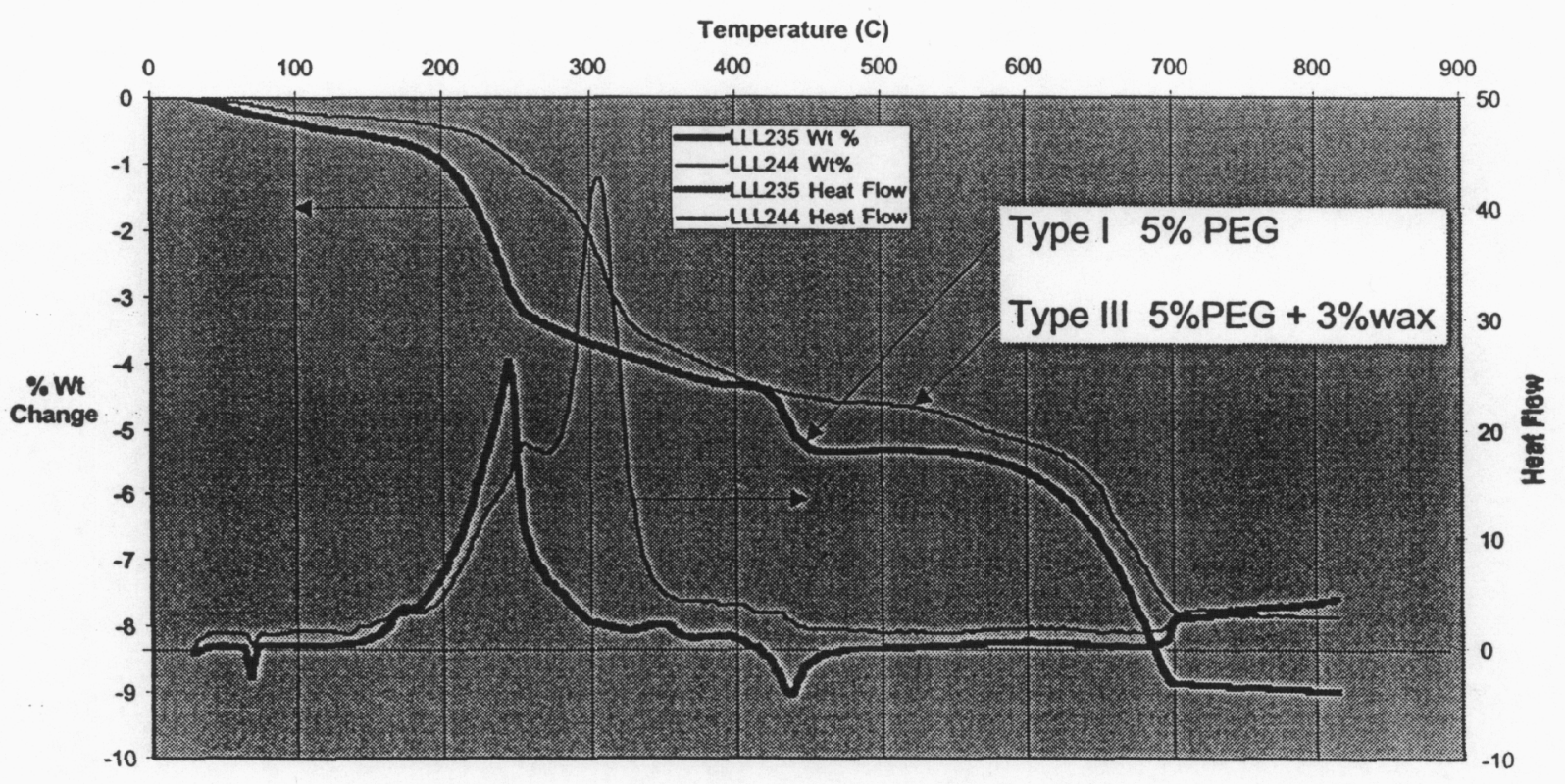

Figure 20. DT/TGA of Type I and III samples 


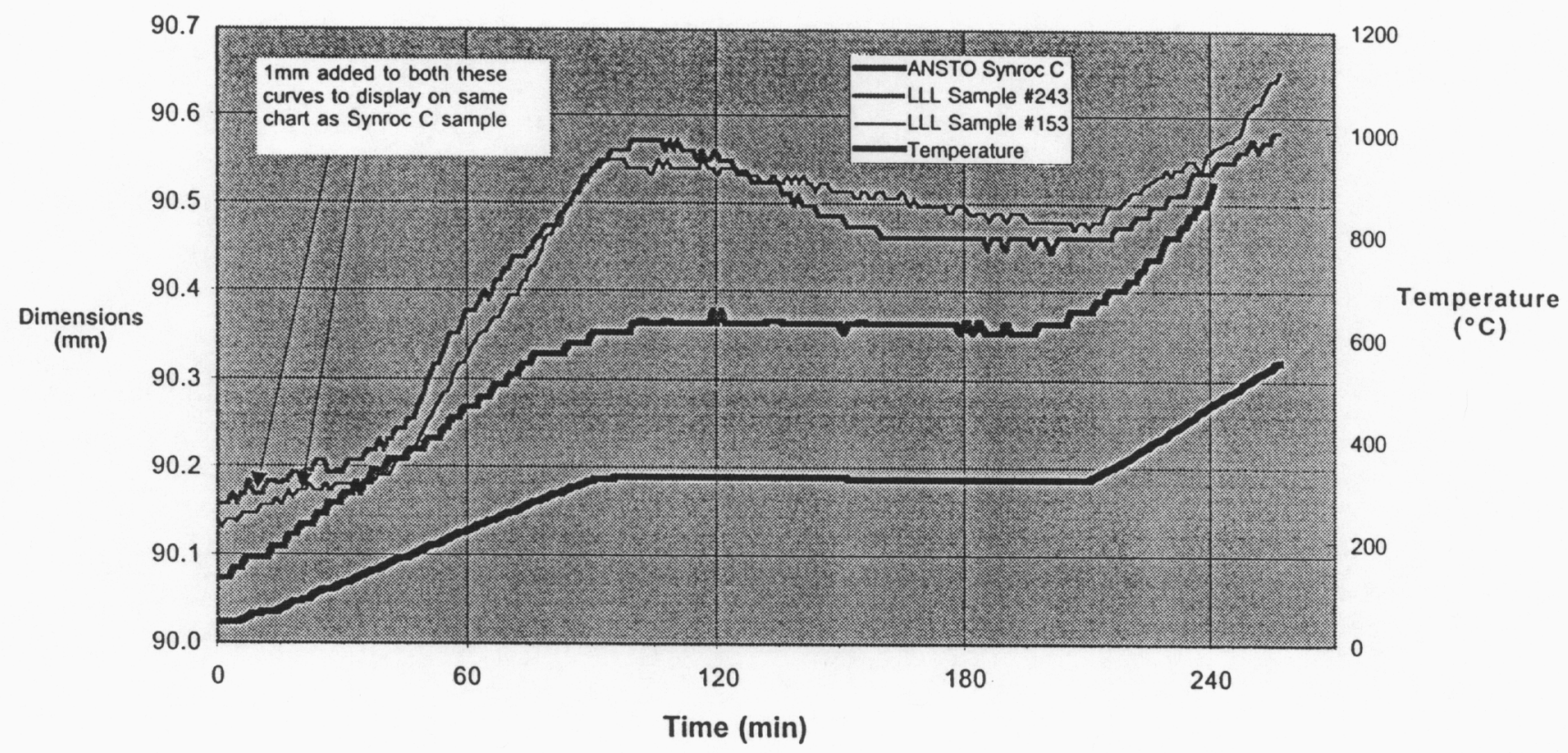

Figure 21 Non-Uniform Dilation in Type I and III samples

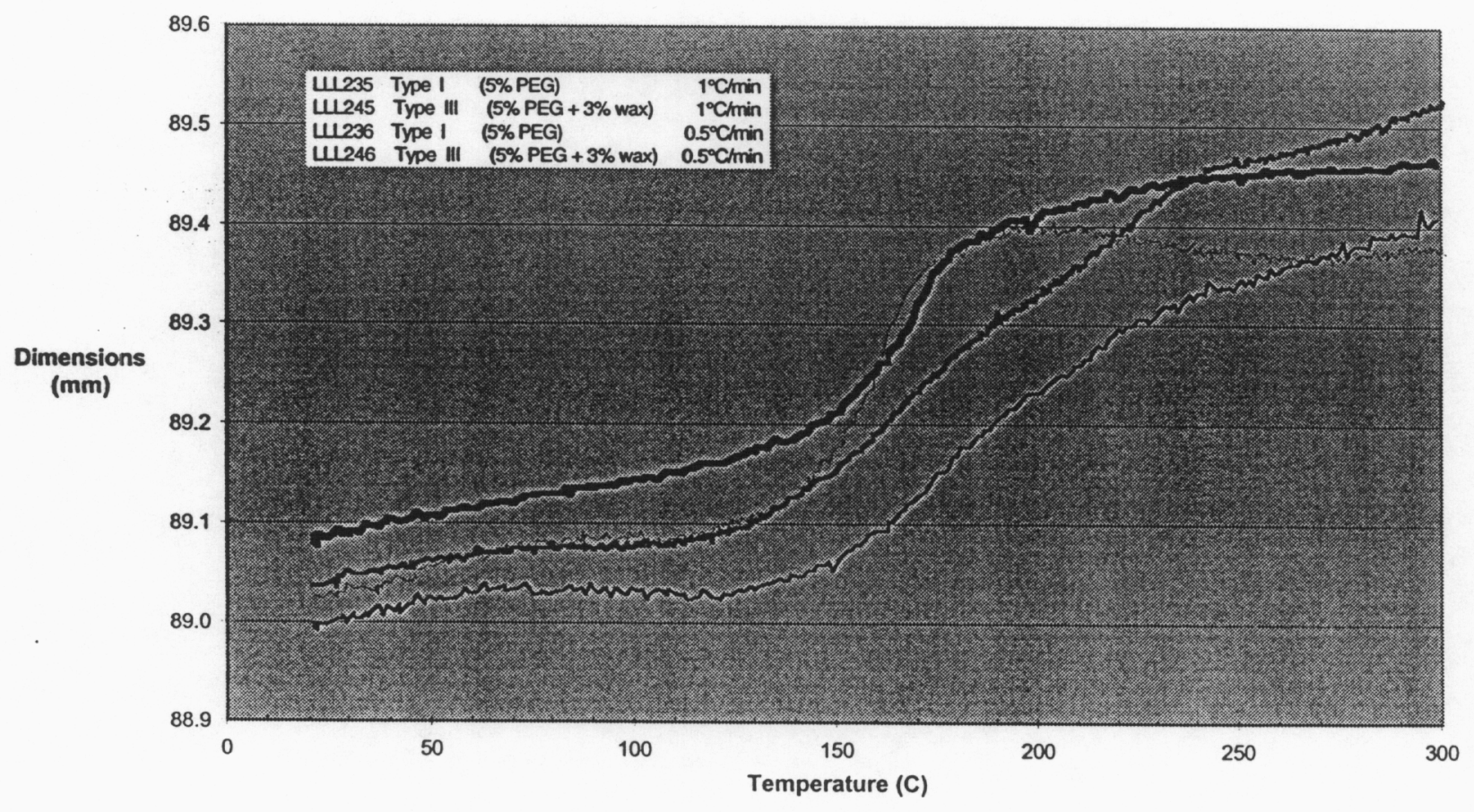

Figure 22 Comparison of Runs SLD 981029 \& SLD 981030 


\section{Analysis of Green Bodies and $300^{\circ} \mathrm{C}$ Heat Treated Samples}

Fracture surfaces of pucks from the $300^{\circ} \mathrm{C}$ heat treated samples are shown in Figure 23 ad. The samples, which were still in their green state, were simply broken into pieces using a hammer.

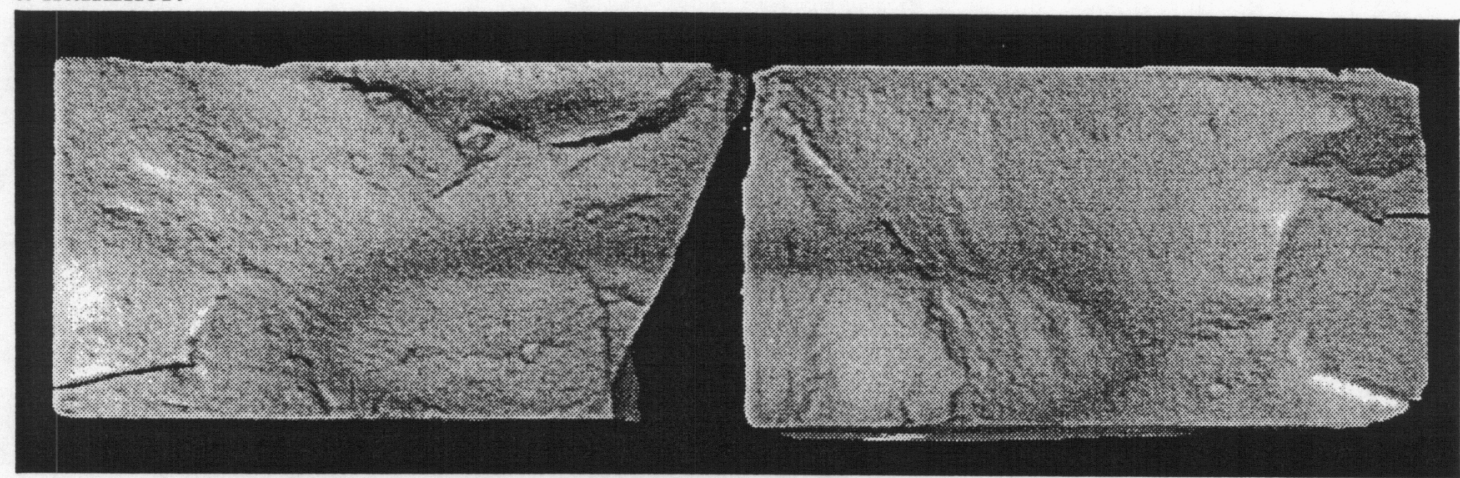

(a) LLL235 Type I Wet Ball Milled, 5\% PEG only $\left(1^{\circ} \mathrm{C} / \mathrm{min}\right.$ to $\left.300^{\circ} \mathrm{C}\right)$

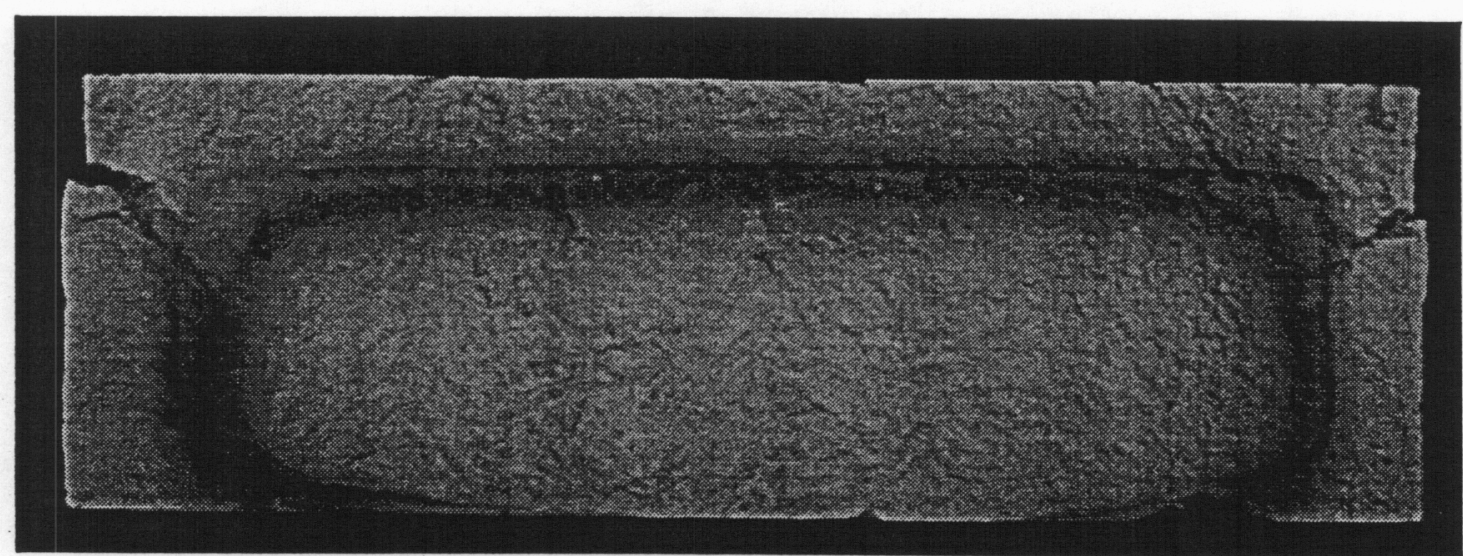

(b) LLL245 Type III Attrition Milled, 5\% PEG + 3\% Wax $\left(1^{\circ} \mathrm{C} / \mathrm{min}\right.$ to $\left.300^{\circ} \mathrm{C}\right)$

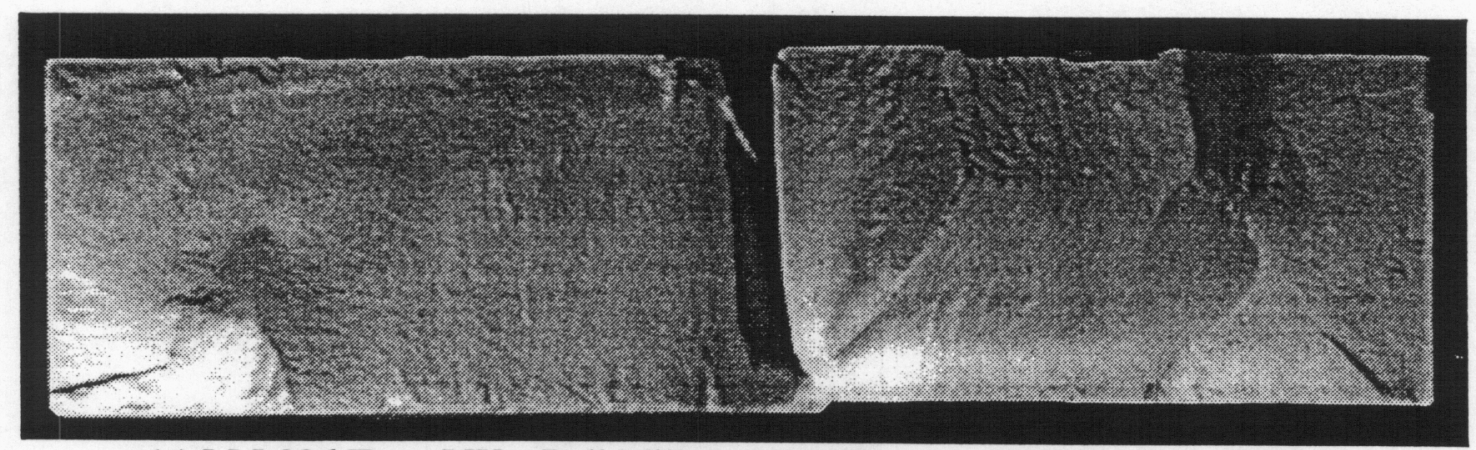

(c) LLL236 Type I Wet Ball Milled, 5\% PEG only $\left(0.5^{\circ} \mathrm{C} / \mathrm{min}\right.$ to $\left.300^{\circ} \mathrm{C}\right)$ 


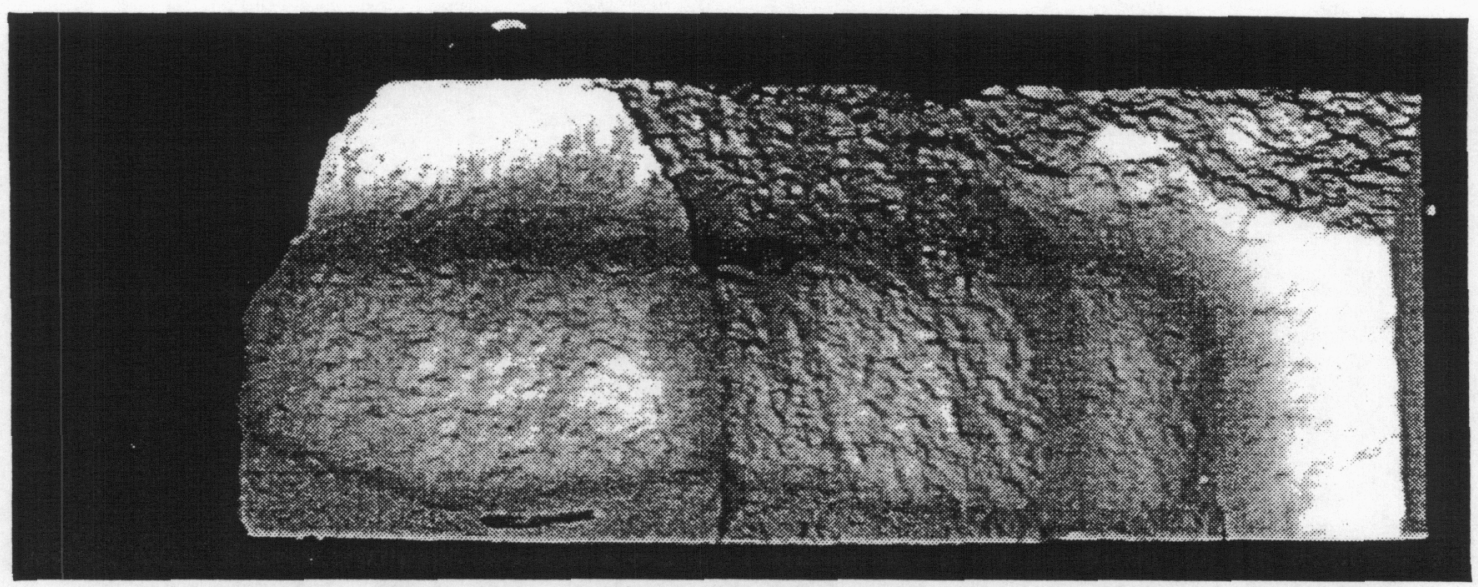

(d) LLL246 Type III Attrition Milled, 5\% PEG + 3\% Wax $\left(0.5^{\circ} \mathrm{C} / \mathrm{min}\right.$ to $\left.300^{\circ} \mathrm{C}\right)$

Figure 23. Fracture surfaces of samples heated to $300^{\circ} \mathrm{C}$ ( $2 \mathrm{hr}$ hold)

The photographs are arranged such that they appear as they were in the furnace, ie. the surface of the sample at the bottom of the photographs was resting on the platinum sheet. The dark regions toward the centre of the sample represent the regions from which the binder and wax components have not fully burned out. NB. Even heating to $300^{\circ} \mathrm{C}$ at $0.5^{\circ} \mathrm{C} / \mathrm{min}$ does not remove all the binder/wax residuals from the sample.

Figures $23 \mathrm{a} \& \mathrm{~b}$ show that the wax component is slower to burn off, see the DT/TGA trace in Figure 20 which supports this statement.

Figure 23c appears to show that all the PEG has been removed from the sample by heating at $0.5^{\circ} \mathrm{C} / \mathrm{min}$ to $300^{\circ} \mathrm{C}$ and holding for 2 hours.

The position of the "unburnt-out " regions is shifted toward the lower surface of the samples. This indicates, either (a) less oxygen can get to the lower face of the sample and/or (b) the platinum sheet acts as a heat sink so the temperature of the sample is lower at its base. This latter suggestion is not realistic as the platinum sheet would conduct heat in from its exposed corners to its centre. This suggests that it would be preferable to raise the pucks from the surface of the setter tile (ie. using coarse powder) or introduce holes into the setter tile to allow oxygen free access to the lower surface of the sample.

The results indicate that a higher burnout temperature should be used, before continuing to sintering temperatures.

Figure 24 shows a cross-section taken through one of the green puck (sample LLL237 Type I material). Inhomogeneities in the distribution of the binder/wax components are readily visible. We are unsure of the exact processing technique, but suggest that the organic components (PEG and wax) should be homogenised with water prior to addition to the ball or attrition mill. An ultrasonic probe should be utilised to create an emulsion of the organics and water. 


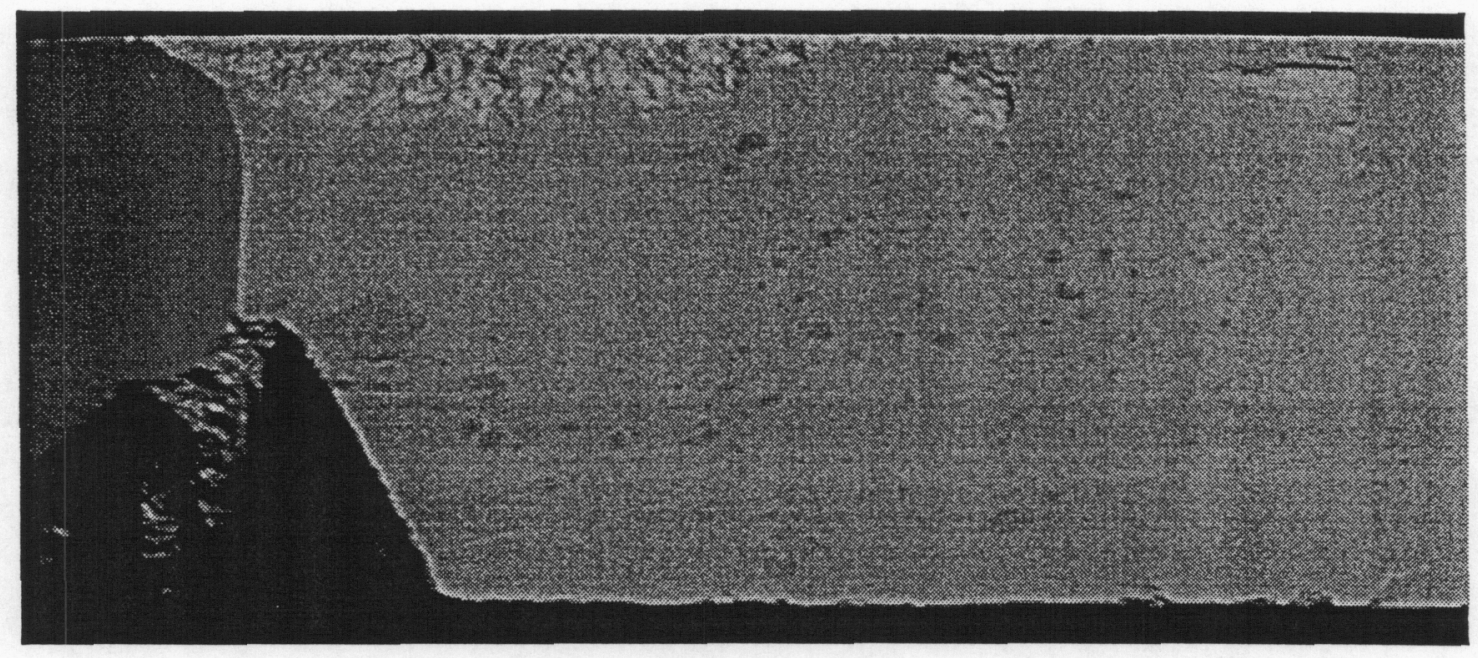

Figure 24 Cross-section of Sample LLL237 Type I, 5\%PEG, Green State.

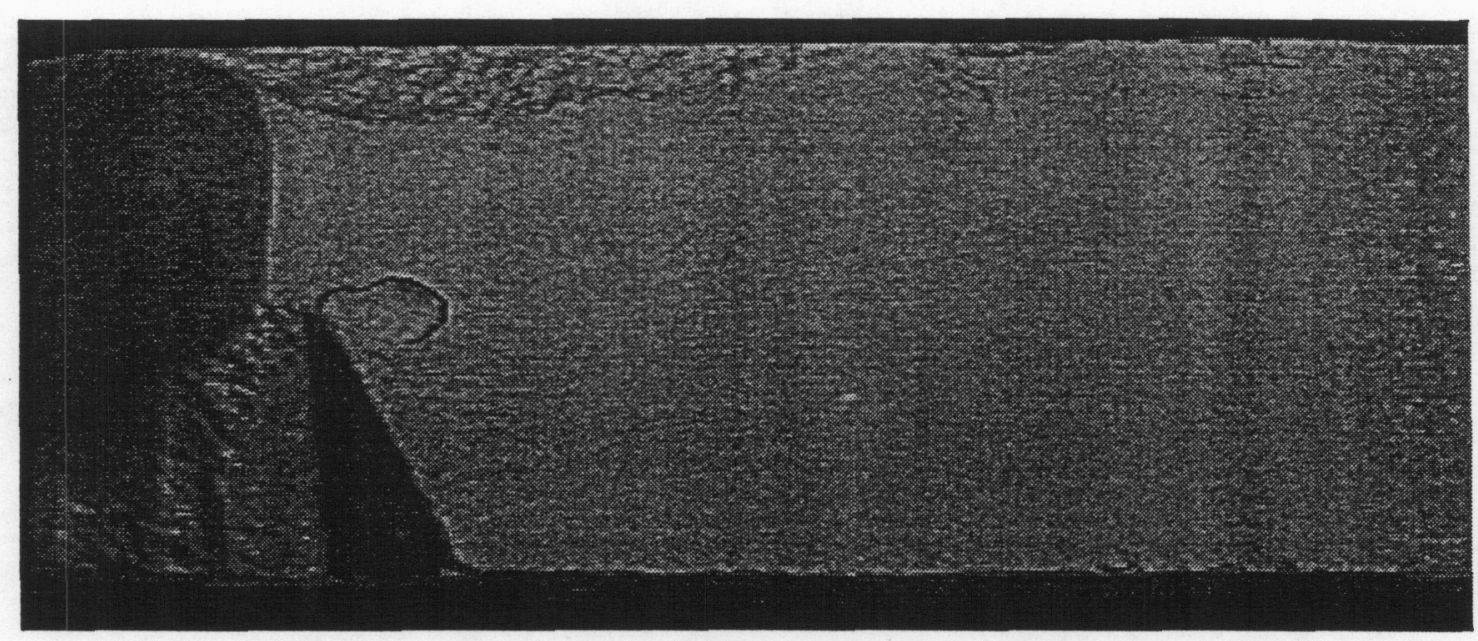

Figure 25 Cross-section of Sample LLL237 Type I, 5\%PEG, Sintered State

Uneven distribution of the binder components can create internal stresses in green compacts. This is the most probable cause of the cracking observed around the large region of organic material (approx. $4 \mathrm{~mm}$ wide by $2 \mathrm{~mm}$ high on the LHS of the sectioned region in Figure 2).

Run SLD A981214 : In this run, a Type II sample was heat-treated using LLLHT005 ie. a heating rate of $3^{\circ} \mathrm{C} / \mathrm{min}$ to $300^{\circ} \mathrm{C}$. The sample was cooled to room temperature after holding for $1 \mathrm{hr}$ at $300^{\circ} \mathrm{C}$. Figure 26 shows the state of the sample after the heat treatment. Cracking occurs in a similar manner to the Type I and III samples. 

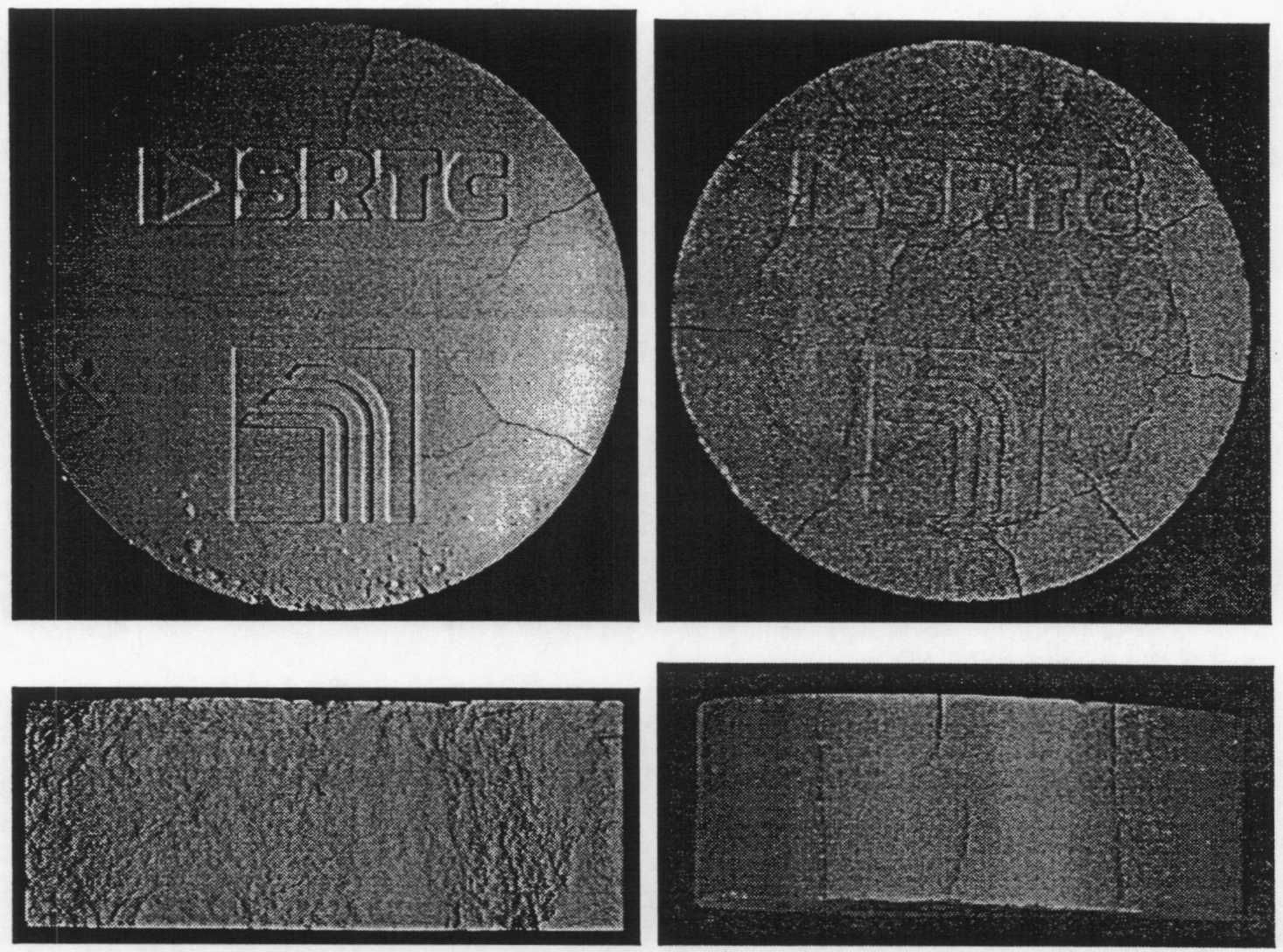

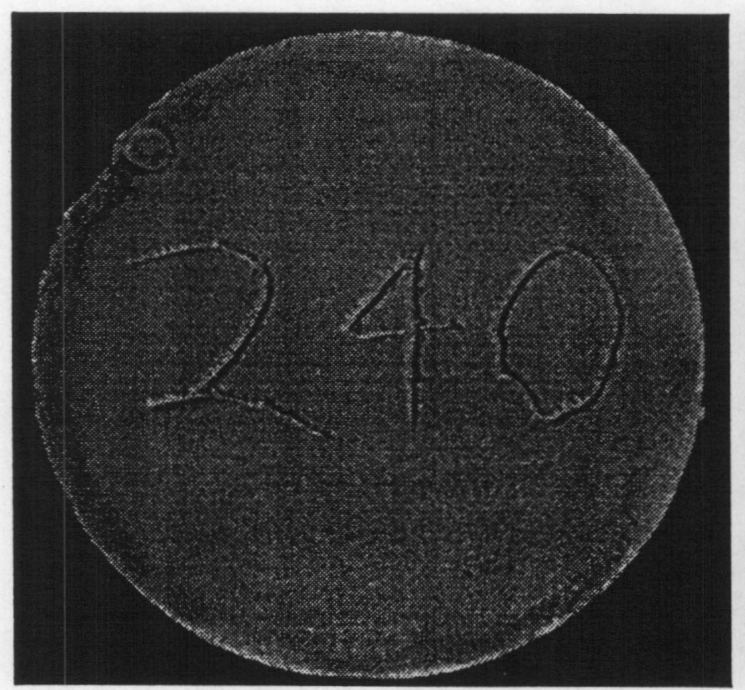

Figure 26

LLL240 Type II $3^{\circ} \mathrm{C} / \mathrm{min}$ to $300^{\circ} \mathrm{C} 1 \mathrm{hr}$

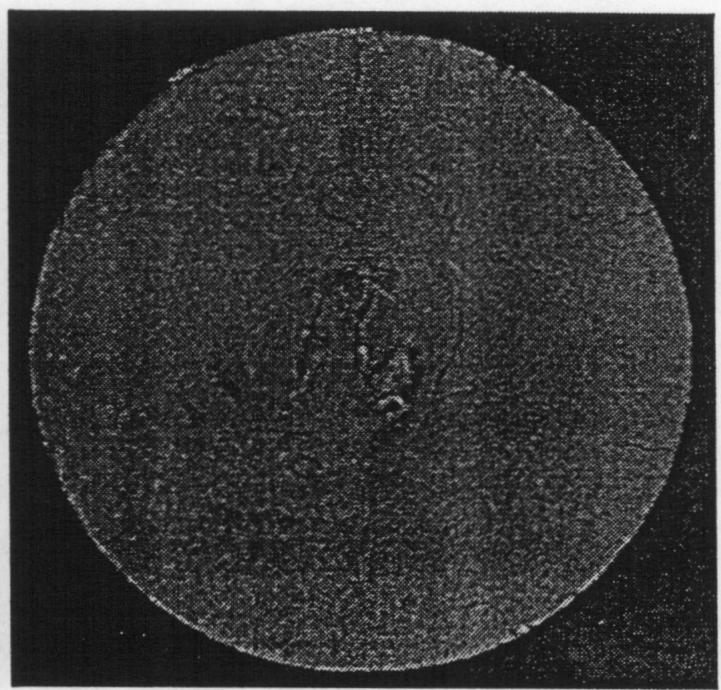

Figure 27 LLL162 Type II $3^{\circ} \mathrm{C} / \mathrm{min}$ to $300^{\circ} \mathrm{C}-1 \mathrm{hr}+$ $5^{\circ} \mathrm{C} / \mathrm{min}$ to $1350^{\circ} \mathrm{C}$ - $4 \mathrm{hr}$

Dr. Philip Walls ANSTO Materials Division 
Run SLD A981215 : The purpose of this run was to prepare a sintered Type II sample for comparison with sintered Type I and III samples. Again cracking was observed.

Run SLD A981217 : This run was performed to examine whether a hold at $110^{\circ} \mathrm{C}$ for 1 hour prior to the binder burnout heat treatment would affect the degree of cracking in the pucks. Sample LLL237 in Figure 28 contains one long crack, (NB the puck broke into two pieces during handling. Sample LLL167, see Figure 29, exhibited similar cracking to the puck that underwent the same type of heat treatment, excluding the $110^{\circ} \mathrm{C}$ hold.

Run SLD A981222 : In this run the green samples LLL 168 \& 239 were CIPed at 200 MPa prior to performing the heat treatment. The change in density of the samples in the green state is shown in Table 6. The CIPing was performed to observe the effect of improving the density of the green compact prior to sintering. As can be seen from the photos in Figures $30 \& 31$, this has exacerbated the degree of cracking. This tends to indicate that the amount of binder / wax is too high, and / or its distribution is insufficiently homogeneous.

Table 6

\begin{tabular}{|c|c|c|c|c|c|}
\cline { 3 - 6 } \multicolumn{2}{c|}{} & \multicolumn{4}{c|}{ Bulk Density } \\
\hline & & \multicolumn{2}{c|}{ As Pressed } & After CIPing @ 200 MPa \\
\hline Sample & Type & g/cc & \% TD & g/cc & \% TD \\
\hline LLL239 & II & 1.99 & 38.3 & 2.76 & 53.1 \\
\hline LLL168 & III & 2.48 & 47.7 & 3.18 & 61.2 \\
\hline \multicolumn{3}{|c|}{ Theoretical Density (TD) assumed to be 5.20 g/cc } \\
\hline
\end{tabular}

\section{Tying the present work in with Task 3.1 Attrition Milling \& Task 3.2 Granulation.}

The laser dilatometer will be used to evaluate powders produced during attrition milling and granulation studies performed at ANSTO in Tasks $3.1 \& 3.2$. We intend to press $\varnothing 90 \mathrm{~mm}$ pellets at ANSTO and evaluate their green properties, including strength and Young's modulus, prior to the sintering studies. 

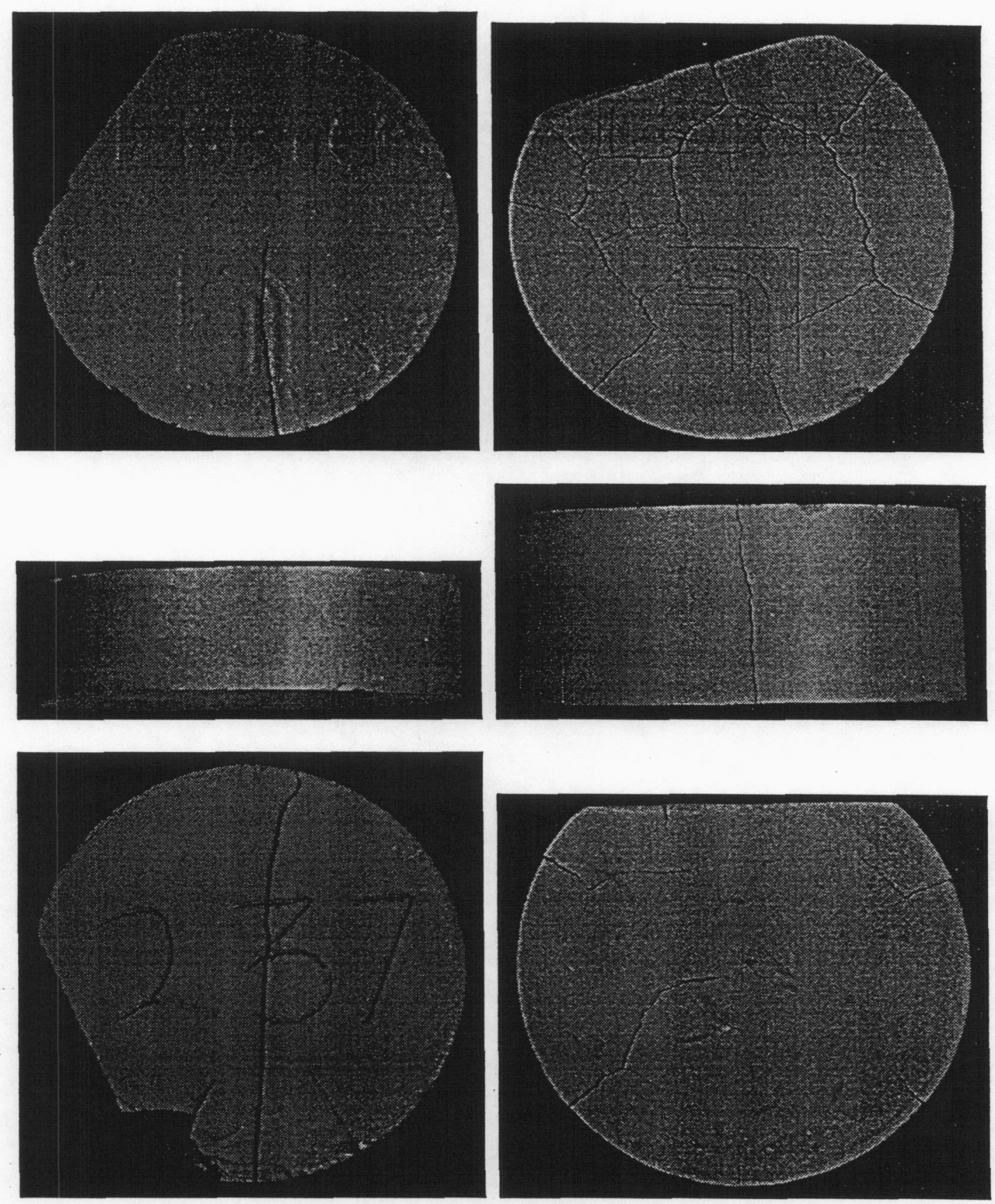

Figure 28

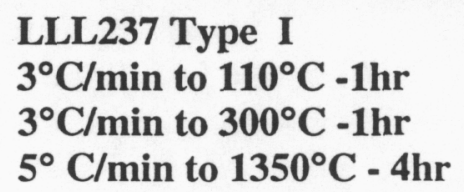

Figure 29

LLL167 Type III $3^{\circ} \mathrm{C} / \mathrm{min}$ to $110^{\circ} \mathrm{C}-1 \mathrm{hr}$ $3^{\circ} \mathrm{C} / \mathrm{min}$ to $300^{\circ} \mathrm{C}-1 \mathrm{hr}$ $5^{\circ} \mathrm{C} / \mathrm{min}$ to $1350^{\circ} \mathrm{C}-4 \mathrm{hr}$

Dr. Philip Walls ANSTO Materials Division 

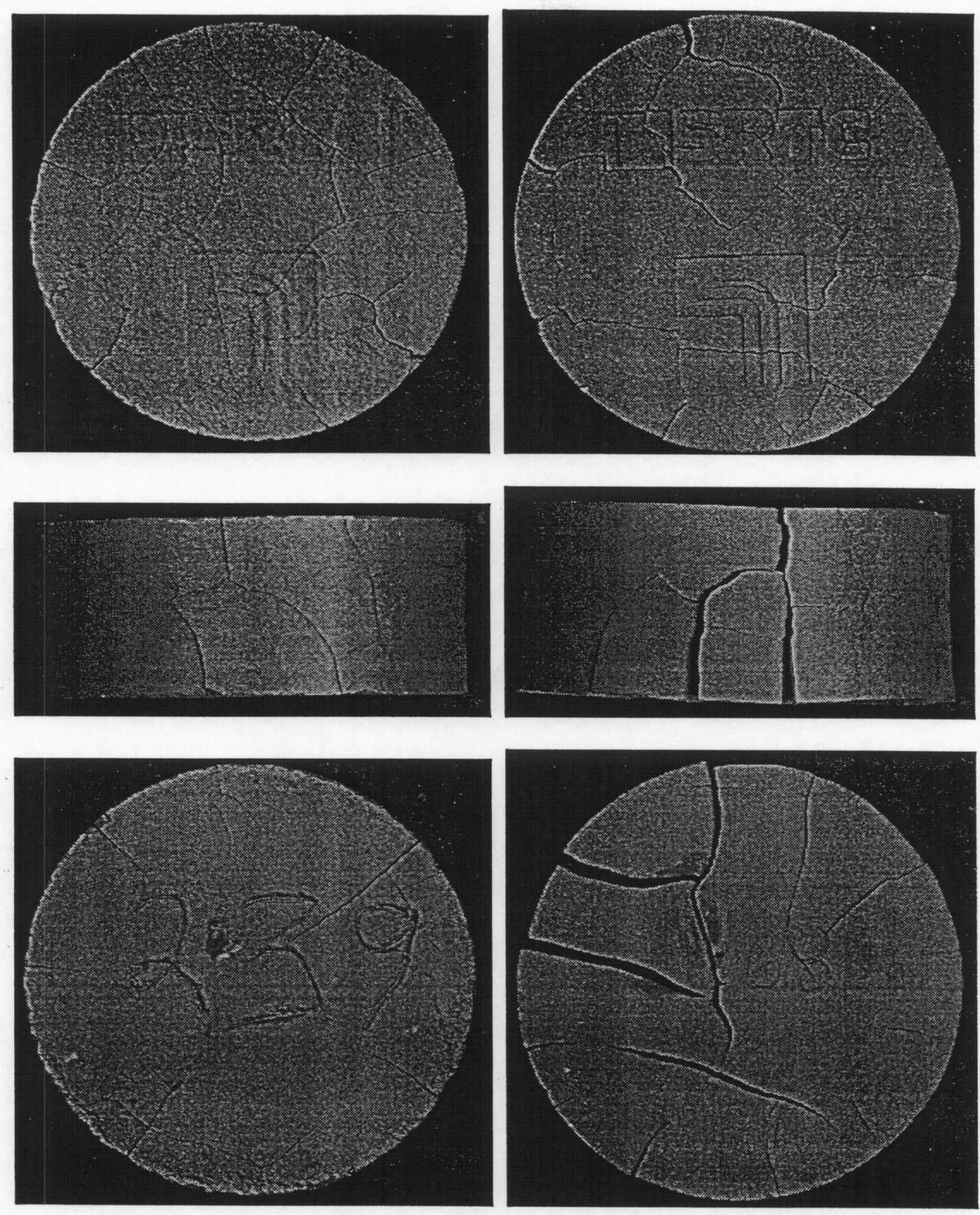

Figure $30 \quad$ LLL239 Type II CIPed @ $200 \mathrm{MPa}$ Heat Treat: LLLHT008

Figure 31

LLL168 Type III CIPed @ $200 \mathrm{MPa}$ Heat Treat: 


\section{Conclusions \& Future Investigations}

1) In the $90 \mathrm{~mm}$ diameter "Puck" samples investigated (Types I, II \& III), cracks occurred in all samples heated to $1350^{\circ} \mathrm{C}$. It was found that these cracks were initiating at low temperatures (between 170 to $300^{\circ} \mathrm{C}$ ) during heat up to the $300^{\circ} \mathrm{C}$ hold.

2) The Laser Dilatometer successfully monitored the cracking process which consisted the following two stages. Stage 1: An increase in the rate of dilation of the sample at approximately $150^{\circ} \mathrm{C}$, ie. an increase in the expansion of the green body in addition to the thermal expansion. This continued to the $300^{\circ} \mathrm{C}$ hold in the heat treatment. Stage 2: Gradual contraction of the sample at the hold temperature. The diameter of the sample was $0.3 \%$ larger than expected from thermal expansion, due to presence of cracks.

3) Cracking was first thought to have been due to pick-up of moisture by the pucks during transportation to and storage at ANSTO. A large increase in the moisture content of the samples was not observed and was discounted as the source of the cracking.

4) Thermogravimetric analysis of small samples taken from the pucks revealed the burnout characteristics of the PEG and polyolefin wax additives. The increase in the rate of dilation of the samples corresponded with initial weight losses from the samples at 150 to $300^{\circ} \mathrm{C}$.

5) Heating to $300^{\circ} \mathrm{C}$ at $1^{\circ} \mathrm{C} / \mathrm{min}$ and even as low as $0.5^{\circ} \mathrm{C} / \mathrm{min}$ did not prevent cracking from occurring, ie. cracking is not a heating rate controlled phenomenon.

6) Although samples were monitored during heating to $1350^{\circ} \mathrm{C}$, as well as during cooling, cracking would have affected both the thermal expansion data and the sintering curves, making comparison of data impossible. Sintering studies should be performed only on pucks, which do not crack.

7) Some decrease in the sintering rate was observed at $1100^{\circ} \mathrm{C}$, which could possibly be due to phase transformations which involve volume expansion.

8) Emulsification of the binder and wax with a solvent prior to adding to the ball or attrition mill is advised. This would improve the distribution of the organic components.

9) Cold isostatic pressing of the green puck samples prior to heat treatment made the cracking phenomenon worse even though the green density had increased significantly. This tends to indicate that the proportion of organic components in the green bodies is too high. 
10) The next stage of work should include optimisation of green forming conditions to allow crack free samples to be produced at relatively fast $\left(3^{\circ} \mathrm{C} / \mathrm{min}\right)$ heating rates to the burnout temperature. The effect of ramp rate to the sintering temperature, sintering temperature and hold time can then be investigated.

11) Further burnout studies should be performed at higher temperatures to remove more of the organic components before semi-rapid heating $\left(\mathrm{eg} .5^{\circ} \mathrm{C} / \mathrm{min}\right)$ to the sintering temperature.

12) Scanning electron microscopy (backscattered mode) investigation of the green bodies will be used to determine the homogeneity of the oxide powders.

13)Distribution of the organic components will be determined using a UV fluorescent dye added during initial ultrasonic homogenisation of the binder, plasticiser and lubricant components with water. After the granulation process, when the organic components will be incorporated into the powder, small pellets will be pressed and examined using optical microscopy using UV illumination.

14) Tying the present work with Task $3.1 \&$ 3.2. ie. using the laser dilatometer to evaluate powders produced by attrition milling at ANSTO: this will involve pressing pellets at ANSTO ( $\varnothing 90 \mathrm{~mm}$ die is on order) and evaluating their green properties, including strength and Youngs modulus, prior to sintering studies. 